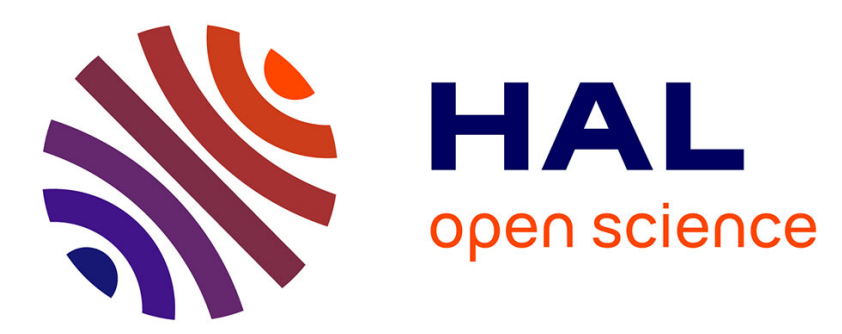

\title{
A bipotential-based limit analysis and homogenization of ductile porous materials with non-associated Drucker-Prager matrix
}

Long Cheng, Yun Jia, Abdelbacet Oueslati, Géry de Saxcé, Djimedo Kondo

\section{- To cite this version:}

Long Cheng, Yun Jia, Abdelbacet Oueslati, Géry de Saxcé, Djimedo Kondo. A bipotential-based limit analysis and homogenization of ductile porous materials with non-associated Drucker-Prager matrix. Journal of the Mechanics and Physics of Solids, 2015, 77, pp.1-26. 10.1016/j.jmps.2014.12.004 . hal02046995

\section{HAL Id: hal-02046995 https://hal.science/hal-02046995}

Submitted on 23 Feb 2019

HAL is a multi-disciplinary open access archive for the deposit and dissemination of scientific research documents, whether they are published or not. The documents may come from teaching and research institutions in France or abroad, or from public or private research centers.
L'archive ouverte pluridisciplinaire HAL, est destinée au dépôt et à la diffusion de documents scientifiques de niveau recherche, publiés ou non, émanant des établissements d'enseignement et de recherche français ou étrangers, des laboratoires publics ou privés. 


\title{
A bipotential-based limit analysis and homogenization of ductile porous materials with non-associated Drucker-Prager matrix
}

\author{
Long Cheng ${ }^{\mathrm{a}}$, Yun Jia ${ }^{\mathrm{a}}$, Abdelbacet Oueslati ${ }^{\mathrm{a}}$, Géry de Saxcéa $^{\mathrm{a}}$, Djimedo Kondo ${ }^{\mathrm{b}}$ \\ ${ }^{a}$ Laboratoire de Mécanique de Lille, UMR CNRS 810\%, Université de Lille 1, Cité scientifique, F59655 \\ Villeneuve d'Ascq, France \\ ${ }^{b}$ Institut Jean Le Rond d'Alembert, UMR CNRS 7190, Université Pierre et Marie Curie, 4 place Jussieu, \\ F75005 Paris, France
}

\begin{abstract}
In Gurson's footsteps, different authors have proposed macroscopic plastic models for porous solid with pressure-sensitive dilatant matrix obeying to the normality law (associated materials). The main objective of the present paper is to extend this class of models to porous materials in the context of non-associated plasticity. This is the case of Drucker-Prager matrix for which the dilatancy angle is different from the friction one, and classical limit analysis theory cannot be applied. For such materials, the second last author has proposed a relevant modeling approach based on the concept of bipotential, a function of both dual variables, the plastic strain rate and stress tensors. On this ground, after recalling the basic elements of the bipotential theory, we present the corresponding variational principles and the extended limit analysis theorems. Then, we formulate a new variational approach for the homogenization of porous porous materials with non-associated matrix. This is implemented by considering the hollow sphere model with a non-associated Drucker-Prager matrix. The proposed procedure delivers a closed form expression of the macroscopic bifunctionnal from which is readily obtained the criterion and a non-associated flow rule of the porous material. It is shown that these general results recover several available models as particular cases. Finally, the established results are assessed and validated by comparing their predictions to that of Finite Element computations carried out on a cell representing the considered class of materials.
\end{abstract}

Keywords: Bipotential theory, Nonlinear Homogenization, Extended Limit analysis, Ductile porous materials, Non associated plasticity, Drucker-Prager matrix 2000 MSC: 74C05, 74L10

\section{Introduction}

In his famous paper, Gurson (1977) proposed an upper bound limit analysis approach of a hollow sphere and a hollow cylinder having a von Mises solid matrix. Several extensions of 
Gurson's model have been further proposed in the literature, the most probably important developments being those accounting for void shape effects (Gologanu et al., 1997; Garajeu et al., 1997; Monchiet et al., 2007; Madou and Leblond, 2012a,b; Monchiet and Kondo , 2013). Plastic anisotropy was treated by (Benzerga et al., 2001; Monchiet et al., 2008; Keralavarma and Benzerga , 2010), while studies by Cazacu et al. (Cazacu and Stewart, 2009) have been devoted to porous materials exhibiting a tension-compression asymmetry. Other extensions take into account the plastic compressibility of the matrix through associated Drucker-Prager model for applications to polymer and cohesive geomaterials (Jeong et al., 1995; Jeong , 2002; Guo et al., 2008; Barthélémy et al., 2003). Application of this class of models has been done in (Lin et al., 2011a,b; Shen et al., 2012). It is worth noticing that, in the spirit of Gurson's paper, the kinematical limit analysis of porous materials with an associated matrix requires the choice of a trial velocity field. The latter is generally built by adding linear terms to the exact one for hydrostatic loading. A notable study concerning the non-associated DruckerPrager matrix has been done by Maghous et al. (2009) in the context of modified secant moduli approach (see also Ponte-Castaneda (1991); Suquet (1995)).

Coming to a more general point of view, a constitutive law in Mechanics is a relationship between dual variables. The constitutive laws of the materials can be represented, as in Elasticity, by a univalued mapping or, as in Plasticity, can be generalized in the form of a multivalued mapping but this representation is not necessarily convenient. When the graph is maximal cyclically monotone, one can model it thanks to a convex and lower semicontinuous function $\pi$, called a superpotential (or pseudo-potential), such that the graph is the one of its subdifferential $\partial \pi$ ). The function $\pi$ and its Fenchel conjugate one $\pi^{*}$ verifies for any couple of dual variables Fenchel's inequality. The dissipative materials admitting a superpotential of dissipation are often qualified as standard (Halphen et al., 1975) and the law is said to be a normality law, a subnormality law or an associated law.

However, many experimental observations in the last decades have motivated the proposition of non-associated laws, particularly in Plasticity theory. For such laws, the second last author proposed in (de Saxcé et al., 1991; de Saxcé , 1992) a suitable modeling framework based on the bipotential, a function $b$ of both dual variables, convex and lower semicontinuous in each argument and satisfying a cornerstone inequality saying that for any couple of dual variables the value of the bipotential is greater than or equal to their duality pairing. When equality holds, the couple is said extremal. In a mechanical point of view, the extremal couples are the ones satisfying the constitutive law. Materials admitting a bipotential are called implicit standard materials (ISM) because the constitutive law is a subnormality law but the relation between the dual variables is implicit. The classical standard materials correspond to the particular event for which the bipotential is separated as the sum of a superpotential and its conjugate one. In this sense, the cornerstone inequality of the bipotential generalizes Fenchel's one. The existence and construction of a bipotential for a given constitutive law has been recently discussed in (Buliga et al., 2008, 2009a, 2010a).

Linked to the structural mechanics and in particular with the Calculus of Variation, the bipotential theory offers an elegant framework to investigate a broad spectrum of nonassociated laws. Examples of such non-associated constitutive laws are: 
- in soil mechanics, non-associated Drucker-Prager (de Saxcé , 1993; Berga et al., 1994; de Saxcé , 1998a; Bousshine et al., 2001; Hjiaj et al., 2003) and Cam-Clay models (de Saxcé , 1995; Zouain et al., 2007, 2010),

- the non linear kinematical hardening rule for cyclic Plasticity (de Saxcé, 1992; Bodovillé et al., 2001) and Viscoplasticity (Hjiaj et al., 2000; Magnier et al., 2006; Bouby et al., 2006, 2009),

- Lemaitre's coupled plasticity-damage law (Bodovillé , 1999),

- the coaxial laws (de Saxcé , 2002; Vallée et al., 2005),

- Coulomb's friction law (de Saxcé , 1998b, 1992, 1993, 1998b,a; Bousshine et al., 2002; Hjiaj et al., 2002, 2004; Feng et al., 2006b,a; Fortin et al., 1999, 2002; Laborde et al., 2008),

- the blurred constitutive laws (Buliga et al., 2009b, 2010b).

A complete survey of the bipotential approach can be found in de Saxcé (2002). In the previous works, robust numerical algorithms were proposed to solve structural mechanics problems.

Coming back to the Limit Analysis let us say that a general method to determine the plastic collapse of structures under proportional loading (Suquet, 1982; Salençon, 1983; Save et al., 1997), even particular in soil mechanics (Chen , 1975; Chen et al., 1990), but it is restricted to associated plasticity, then with normality law. The classical presentation of the non-associated plasticity is based on a yield function and a plastic potential. The bipotential offers an alternative formulation which naturally opening to a variational formulation, and then paving the way for an extension of limit analysis techniques to non-associated laws (de Saxcé , 1998a; Bousshine et al., 2001, 2002; Chaaba et al., 2010; Zouain et al., 2007). Extension of limit analysis theory to the repeated variable loading, known as shakedown theory, has been successfully generalized to the ISM $^{1}$ by the bipotential approach in (de Saxcé , 2002; Bousshine et al., 2001, 2003; Bouby et al., 2006, 2009).

The aim of the present study is to formulate a macroscopic model for "ductile porous materials with a non-associated Drucker-Prager"-type matrix, using homogenization techniques combined with the bipotential theory. The paper is organized as follows: the non-associated Drucker-Prager plastic model, for which the yield criterion and plastic potential are respectively defined by two functions, is first summarized in Section 2. Next, we introduce in Section 3, the bipotential theory and its two dual fields (stress and velocity fields) based formulation, which allows us to derive the plastic criterion and the non-associated flow rule. An application of the bipotential theory to the non-associated Drucker-Prager plastic model is particularly discussed in subsection 3.3. Section 4 is devoted to the bipotential-based extended limit analysis approach of non-associated porous media. The proposed formulation

\footnotetext{
${ }^{1}$ See also the use of Shakedown theory by (Boulbibane and Weichert, 1997) for non-associated soils.
} 
provides a fundamental variational theory for the macroscopic modeling of a large class of porous media. In Section 5, the proposed bipotential-based theory is implemented in the case of a hollow sphere having a rigid perfectly plastic matrix obeying to a non-associated Drucker-Prager flow rule. This implementation will be performed by adopting simple trial stress and velocity fields. This allows to derive in subsection 5.3 a closed-form expression of the macroscopic criterion and the non-associated plastic flow rule. Furthermore, some special cases, corresponding to existing models previously proposed in literature, are discussed in subsection 6. Finally, in Section 7, the established macroscopic criterion, flow rule and void evolution are respectively assessed and validated by comparison with Finite Element solutions.

\section{Brief recall the non-associated Drucker-Prager model}

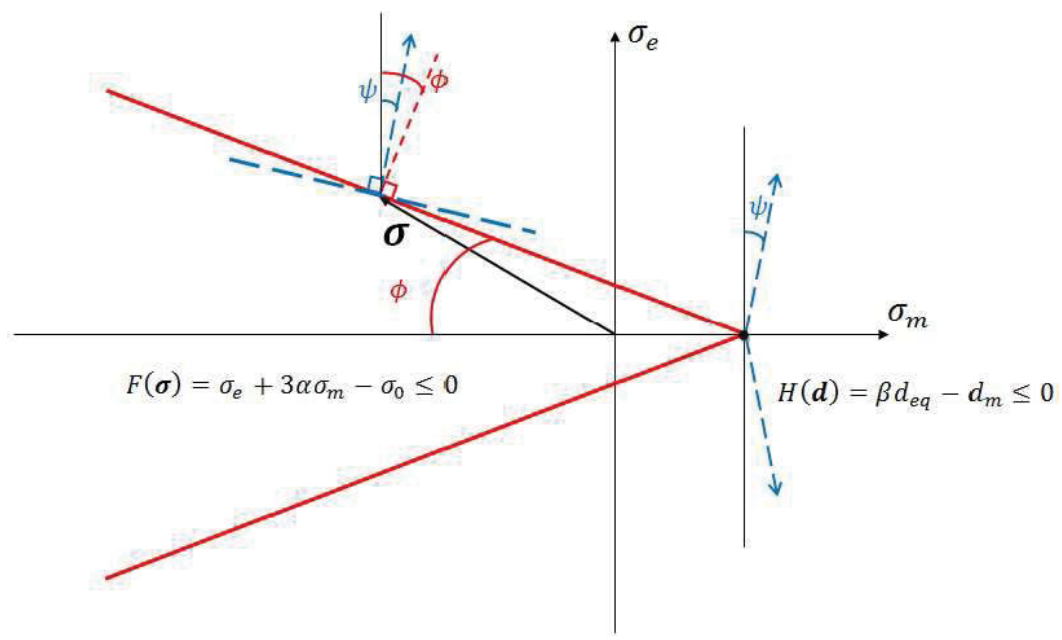

Figure 1: Drucker-Prager model: yield criterion and non-associated flow rule

Drucker-Prager model (Fig.1) requires the consideration of a yield criterion in the form:

$$
F(\boldsymbol{\sigma})=\sigma_{e}+3 \alpha \sigma_{m}-\sigma_{0} \leq 0
$$

where $\sigma_{e}$ is the equivalent stress, $\sigma_{m}$ the mean stress, $\sigma_{0}>0$ the shear cohesion stress of the material and $\alpha$ the pressure sensitivity factor related to the friction angle $\phi$ by:

$$
\tan \phi=3 \alpha .
$$

Let us introduce the plastic potential:

$$
G(\boldsymbol{\sigma})=\sigma_{e}+3 \beta \sigma_{m}
$$

where $\beta(\beta \leq \alpha)$ depends on the dilatancy angle $\psi$ through:

$$
\tan \psi=3 \beta \text {. }
$$


Except for the apex of Drucker-Prager cone $\left(\sigma_{e}=0, \sigma_{m}=\sigma_{0} / 3 \alpha\right)$ where $\sigma_{e}$ is not differentiable, the plastic strain rate is given by the non-associated yielding rule:

$$
\boldsymbol{d}=d_{e q} \frac{\partial G}{\partial \boldsymbol{\sigma}}=d_{e q}\left(\frac{3 \boldsymbol{s}}{2 \sigma_{e}}+\beta \mathbf{1}\right)
$$

where $\boldsymbol{\sigma}$ is Cauchy stress tensor, $\boldsymbol{s}$ the deviatoric stress, $\mathbf{1}$ the unit tensor. $d_{e q}=\left|\frac{2}{3} \boldsymbol{d}^{\prime}: \boldsymbol{d}^{\prime}\right|^{1 / 2}$ with $\boldsymbol{d}^{\prime}$ being the deviatoric part of $\boldsymbol{d}$. The plastic dilatancy reads:

$$
d_{m}=\frac{1}{3} \operatorname{tr} \boldsymbol{d}=\beta d_{e q}
$$

This suggests to introduce:

$$
H(\boldsymbol{d})=\beta d_{e q}-d_{m}
$$

The plastic yielding rule (3) is completed at the apex by the admissibility condition:

$$
H(\boldsymbol{d}) \leq 0
$$

while, because of $(4), H(\boldsymbol{d})=0$ at the other points of the yielding surface (called regular points). Of course, for the particular event $\psi=\phi$, the normality rule is recovered and the plasticity model is associated. Without loss of generality, we can assume that:

$$
0 \leq \beta \leq \alpha<\frac{1}{2}
$$

or equivalently $0 \leq \psi \leq \phi<56^{\circ} 18^{\prime}$. In practice, these conditions are fulfilled by the geomaterials and other pressure sensitive dilatant materials. Some examples of experimental data concerning the friction angle can be found for polymers, high strength steels and aluminium in (Guo et al., 2008).

\section{Bipotential-based formulation of constitutive models}

Rigid perfectly plastic model is usually considered to obtain the analytical solution (plastic criterion and potential) for a large class of materials, simultaneously by adopting the Limit Analysis approach, which is extensively discussed in literature . However, this conventional approach can only be rigorously used for Generalized Standard Materials (GSM), that is materials which obey a normality law. the standard limit analysis framework is then not suitable for materials which obey to a non-associated flow rule (for instance, geomaterials). This question has been discussed in several works (Salençon , 1983; Drucker , 1953; Palmer , 1973; Radenkovic , 1961; Telega , 2002; Telega et al. , 2004).

In order to overcome this problem, de Saxcé et al. has proposed in previous papers a new modeling of the non-associated constitutive laws based on the concept of bipotential (de Saxcé et al., 1991; de Saxcé , 1992). 


\subsection{The bipotential in short}

First of all, let us recall a basic concept of convex analysis, the subdifferential of a function $\pi$ in a point $\boldsymbol{x}$ which is the (possibly empty) set:

$$
\partial \pi(\boldsymbol{x})=\left\{\boldsymbol{y} \mid \forall \boldsymbol{x}^{\prime}, \quad \pi\left(\boldsymbol{x}^{\prime}\right)-\pi(\boldsymbol{x}) \geq\left(\boldsymbol{x}^{\prime}-\boldsymbol{x}\right): \boldsymbol{y}\right\} .
$$

For more details on convex analysis, the reader is refered for instance to (Ekeland et al., 1975; Moreau , 2003; Rockafellar , 1970). Moreover, in mechanics, GSM can represented as a generalized model based on two superpotentials $\pi\left(\boldsymbol{x}^{\prime}\right)$ and $\pi^{*}\left(\boldsymbol{y}^{\prime}\right)$, which are depending on a represented strain rate variable $\boldsymbol{x}$ and a stress-like one $\boldsymbol{y}$. Such a couple of superpotentials satisfies the Fenchel's inequality (Fenchel , 1949),

$$
\forall\left(\boldsymbol{x}^{\prime}, \boldsymbol{y}^{\prime}\right) \quad \pi\left(\boldsymbol{x}^{\prime}\right)+\pi^{*}\left(\boldsymbol{y}^{\prime}\right) \geq \boldsymbol{x}^{\prime}: \boldsymbol{y}^{\prime}
$$

where $\pi\left(\boldsymbol{x}^{\prime}\right)$ and $\pi^{*}\left(\boldsymbol{y}^{\prime}\right)$ are convex, lower semicontinuous and conjugate each of the other. The r.h.s. of (7) indicates the inner product of $\boldsymbol{x}^{\prime}$ and $\boldsymbol{y}^{\prime}$. When the equality is achieved, $(\boldsymbol{x}, \boldsymbol{y})$ is called an extremal couple:

$$
\pi(\boldsymbol{x})+\pi^{*}(\boldsymbol{y})=\boldsymbol{x}: \boldsymbol{y}
$$

It can be proved that this relation is equivalent to the two following differential inclusions:

$$
\begin{gathered}
\boldsymbol{y} \in \partial \pi(\boldsymbol{x}), \\
\boldsymbol{x} \in \partial \pi^{*}(\boldsymbol{y}) .
\end{gathered}
$$

It is worth to remark that the convexity properties of $\pi$ and $\pi^{*}$ are essential in order to state and prove minimum variational principles and use the limit analysis approach ${ }^{2}$. When the normality law fails and is replaced by a non-associated flow rule, the classical presentation is based on a yield function (to model the yield criterion) and a plastic potential (to represent the flow rule). Although it is intensively used in the literature, this is, in fact, not very relevant for the variational methods. On the ground of this observation, de Saxcé and collaborators proposed in (de Saxcé et al., 1991; de Saxcé , 1992) a suitable modeling based on more general generating functions called bipotentials and defined by the following properties:

(a) $b$ is convex and lower semicontinuous in each argument.

(b) For any $\boldsymbol{x}^{\prime}$ and $\boldsymbol{y}^{\prime}$ we have

$$
b\left(\boldsymbol{x}^{\prime}, \boldsymbol{y}^{\prime}\right) \geq \boldsymbol{x}^{\prime}: \boldsymbol{y}^{\prime}
$$

(c) For $\boldsymbol{x}$ and $\boldsymbol{y}$ we have the equivalences:

$$
\mathbf{y} \in \partial b(\cdot, \boldsymbol{y})(\boldsymbol{x}) \Longleftrightarrow \boldsymbol{x} \in \partial b(\boldsymbol{x}, \cdot)(\boldsymbol{y}) \Longleftrightarrow b(\boldsymbol{x}, \boldsymbol{y})=\boldsymbol{x}: \boldsymbol{y}
$$

In a mechanical point of view, the bipotential represents the plastic dissipation power (by volume unit) and (9) is the constitutive law. The couples $(\boldsymbol{x}, \boldsymbol{y})$ for which ones equivalence (9) holds are called extremal couples. The cornerstone inequality (8) clearly generalizes Fenchel's one (7).

\footnotetext{
${ }^{2}$ Lower and upper bound solutions obtained from the Hill's principle and Markov's one.
} 


\subsection{Variational framework of bipotential-based formulations for constitutive laws}

Let us now replace the above notations $\boldsymbol{x}$ and $\boldsymbol{y}$ respectively by the strain rate tensor $\boldsymbol{d}$ and the stress tensor $\boldsymbol{\sigma}$. In a mechanical point of view, the corresponding bipotential represents the plastic dissipation power (by volume unit) and from Eq.(9) the constitutive law can be obtained. Accounting for the definition (6) of the subdifferential and the cornerstone inequality (8), the constitutive law reads (Buliga et al., 2009a, 2010a; Laborde et al., 2008; Buliga et al., 2010b):

$$
\min _{\boldsymbol{d}^{\prime}}\left(b\left(\boldsymbol{d}^{\prime}, \boldsymbol{\sigma}\right)-\boldsymbol{d}^{\prime}: \boldsymbol{\sigma}\right)=\min _{\boldsymbol{\sigma}^{\prime}}\left(b\left(\boldsymbol{d}, \boldsymbol{\sigma}^{\prime}\right)-\boldsymbol{d}: \boldsymbol{\sigma}^{\prime}\right)=0 .
$$

It is worth remarking that, with respect to the previous minimization problems, the bipotential has the required convexity properties.

Next, let us show how to recover simply the plastic yielding condition $F(\boldsymbol{\sigma})=0$ by the bipotential formalism. To this end, the first minimization problem in (10) becomes:

$$
\min _{H(\boldsymbol{d}) \leq 0}\left(b_{0}(\boldsymbol{d}, \boldsymbol{\sigma})-\boldsymbol{d}: \boldsymbol{\sigma}\right)=0,
$$

where $b_{0}$ is the finite part of the bipotential when the extremal value is taken. Relaxing the kinematical condition $H(\boldsymbol{d}) \leq 0$ by use of Lagrange's multiplier $\lambda$, this constrained minimization problem is transformed into an equivalent saddle-point problem

$$
\max _{\lambda \geq 0} \min _{\boldsymbol{d}}\left(L(\boldsymbol{d}, \boldsymbol{\sigma}, \lambda)=b_{0}(\boldsymbol{d}, \boldsymbol{\sigma})-\boldsymbol{d}: \boldsymbol{\sigma}+\lambda H(\boldsymbol{d})\right)=0,
$$

where $L(\boldsymbol{d}, \boldsymbol{\sigma}, \lambda)$ is the lagrangian function. Its stationarity with respect to $\boldsymbol{d}$ :

$$
\frac{\partial L}{\partial \boldsymbol{d}}=\frac{\partial b_{0}}{\partial \boldsymbol{d}}(\boldsymbol{\sigma})-\boldsymbol{\sigma}+\lambda \frac{\partial H(\boldsymbol{d})}{\partial \boldsymbol{d}}=0
$$

Eliminating the lagrangian multiplier $\lambda$ in above system of equations, the resultant functional depends only on stress tensor $\boldsymbol{\sigma}$. Let us denote it $F$; it follows the yield criterion

$$
F(\boldsymbol{\sigma})=0
$$

In a similar way, it is possible to recover the plastic flow rule (3) at a regular point. The second minimization problem in (10) becomes:

$$
\min _{F(\boldsymbol{\sigma}) \leq 0}\left(b_{0}(\boldsymbol{d}, \boldsymbol{\sigma})-\boldsymbol{d}: \boldsymbol{\sigma}\right)=0 \text {. }
$$

Relaxing the plastic yielding condition $F(\boldsymbol{\sigma}) \leq 0$ by use of Lagrange's multiplier $\lambda^{*}$, this problem is transformed into an equivalent saddle-point problem

$$
\max _{\lambda^{*} \geq 0} \min _{\boldsymbol{\sigma}}\left(L^{*}\left(\boldsymbol{d}, \boldsymbol{\sigma}, \lambda^{*}\right)=b_{0}(\boldsymbol{d}, \boldsymbol{\sigma})-\boldsymbol{d}: \boldsymbol{\sigma}+\lambda^{*} F(\boldsymbol{\sigma})\right)=0,
$$

By calculating the stationarity of the lagrangian $L^{*}\left(\boldsymbol{d}, \boldsymbol{\sigma}, \lambda^{*}\right)$ with respect to $\boldsymbol{\sigma}$

$$
\frac{\partial L}{\partial \boldsymbol{\sigma}}=\frac{\partial b_{0}}{\partial \boldsymbol{\sigma}}(\boldsymbol{d})-\boldsymbol{d}+\lambda^{*} \frac{\partial F(\boldsymbol{\sigma})}{\partial \boldsymbol{\sigma}}=0
$$

and eliminating $\lambda^{*}$, the resultant functional (denoted $H$ ) depends only on the strain rate tensor $\boldsymbol{d}$. Hence, the flow rule can be obtained

$$
H(\boldsymbol{d})=0
$$




\subsection{Case of the non-associated Drucker-Prager materials}

The finite value bipotential of non-associated Drucker-Prager model (see Section 2) takes the form (Hjiaj et al., 2003):

$$
b(\boldsymbol{d}, \boldsymbol{\sigma})=\left\{\begin{array}{c}
\frac{\sigma_{0}}{\alpha} d_{m}+(\beta-\alpha)\left(3 \sigma_{m}-\frac{\sigma_{0}}{\alpha}\right) d_{e q} \quad \text { if } F(\boldsymbol{\sigma}) \leq 0 \quad \text { and } \quad H(\boldsymbol{d}) \leq 0 \\
+\infty \text { otherwise }
\end{array}\right\},
$$

In view of what will be done in subsections 5.2 and 5.3, for the homogenization problem, it is convenient to indicate how the derivation of the non-associated yield criterion can be done from (12) and (14). The lagrangian function reads:

$$
L(\boldsymbol{d}, \boldsymbol{\sigma}, \lambda)=\frac{\sigma_{0}}{\alpha} d_{m}+(\beta-\alpha)\left(3 \sigma_{m}-\frac{\sigma_{0}}{\alpha}\right) d_{e q}-\left(\sigma_{e} d_{e q}+3 d_{m} \sigma_{m}\right)+\lambda\left(\beta d_{e q}-d_{m}\right) .
$$

Its stationnarity with respect to $d_{e q}$ and $d_{m}$ gives:

$$
\begin{gathered}
\sigma_{e}=(\beta-\alpha)\left(3 \sigma_{m}-\frac{\sigma_{0}}{\alpha}\right)+\beta \lambda, \\
3 \sigma_{m}=\frac{\sigma_{0}}{\alpha}-\lambda .
\end{gathered}
$$

Eliminating $\lambda$ between these relations leads to the plastic criterion :

$$
F(\boldsymbol{\sigma})=\sigma_{e}+3 \alpha \sigma_{m}-\sigma_{0}=0 .
$$

Simultaneously, from the second minimization problem of (10), we have the corresponding lagrangian by introducing the multiplier $\lambda^{*}$

$$
L^{*}\left(\boldsymbol{d}, \boldsymbol{\sigma}, \lambda^{*}\right)=\frac{\sigma_{0}}{\alpha} d_{m}+(\beta-\alpha)\left(3 \sigma_{m}-\frac{\sigma_{0}}{\alpha}\right) d_{e q}-\left(\boldsymbol{d}^{\prime}: \boldsymbol{s}+3 d_{m} \sigma_{m}\right)+\lambda^{*}\left(\sigma_{e}+3 \alpha \sigma_{m}-\sigma_{0}\right) .
$$

In the same way, the stationnarity with respect to $s$ and $\sigma_{m}$ reads,

$$
\begin{gathered}
\boldsymbol{d}^{\prime}=\lambda^{*} \frac{3 \boldsymbol{s}}{2 \sigma_{e}}, \\
(\beta-\alpha) d_{e q}-d_{m}+\alpha \lambda^{*}=0 .
\end{gathered}
$$

From (15) one obtains $\lambda^{*}=d_{e q}$. Eliminating $\lambda^{*}$ in (16) leads to the kinematical condition at the regular points upon the yield surface:

$$
H(\boldsymbol{d})=\beta d_{e q}-d_{m}=0,
$$

That allows to recover the non-associated yielding rule (3):

$$
\boldsymbol{d}=\boldsymbol{d}^{\prime}+d_{m} \mathbf{1}=d_{e q}\left(\frac{3 \boldsymbol{s}}{2 \sigma_{e}}+\beta \mathbf{1}\right) .
$$

For the treatment of the apex, the reader is refered to (Hjiaj et al., 2003), which is specifically devoted to this question. 


\section{Extended limit analysis of porous materials with a non-associated matrix}

Unlike the classical presentation of the non-associated constitutive laws by means of the yield function and the plastic potential, the bipotential formulation naturally opens into a variational formulation; this is crucial for an extension of limit analysis techniques to the context of non-associated laws. We present here the main elements of this variational framework in the context of porous media.

\subsection{Determination of the macroscopic bifunctional and its variational properties}

This presentation is directly done in the framework of homogenization of porous material, considering a reference cell $\Omega$ composed of a void $\omega$ and a matrix $\Omega_{M}=\Omega-\omega$ made of an Implicit Standard Material. The macro-cell $\Omega$ is enclosed by surface $\partial \Omega$ and the void $\omega$ by $\partial \omega$. The external boundary of the cell is subjected to a uniform strain rate: $\boldsymbol{v}=\boldsymbol{D} \cdot \boldsymbol{x}, \boldsymbol{x}$ being the position vector at the boundary. The macroscopic stress $\boldsymbol{\Sigma}$ and strain rate $\boldsymbol{D}$ are then classically defined as volume averages of their microscopic counterpart $\boldsymbol{\sigma}$ and $\boldsymbol{d}$ :

$$
\boldsymbol{\Sigma}=\frac{1}{|\Omega|} \int_{\Omega} \boldsymbol{\sigma} d V, \quad \boldsymbol{D}=\frac{1}{|\Omega|} \int_{\Omega} \boldsymbol{d} d V
$$

Note that the set of kinematical admissible velocity fields is defined in the following sense:

$$
\mathcal{K}_{a}=\{\boldsymbol{v} \quad \text { s.t. } \quad \boldsymbol{v}(\boldsymbol{x})=\boldsymbol{D} . \boldsymbol{x} \quad \text { on } \quad \partial \Omega\} .
$$

and the associated strain rate field is given by $\boldsymbol{d}(\boldsymbol{v})=\operatorname{grad}_{s} \boldsymbol{v}=\frac{1}{2}\left(\operatorname{grad}_{\boldsymbol{v}}+\operatorname{grad}^{T} \boldsymbol{v}\right)$.

The set of statically admissible stress fields is:

$$
\mathcal{S}_{a}=\left\{\boldsymbol{\sigma} \quad \text { s.t. } \quad \operatorname{div} \boldsymbol{\sigma}=0 \quad \text { in } \quad \Omega_{M}, \quad \boldsymbol{\sigma} \cdot \boldsymbol{n}=0 \quad \text { on } \quad \partial \omega, \quad \boldsymbol{\sigma}=0 \quad \text { in } \quad \omega\right\} .
$$

The set of admissible couples is the product $\mathcal{A}=\mathcal{K}_{a} \times \mathcal{S}_{a}$ and the set of extremal ones is defined by:

$$
\mathcal{E}=\left\{(\boldsymbol{v}, \boldsymbol{\sigma}) \quad \text { s.t. } \quad(\boldsymbol{d}(\boldsymbol{v}), \boldsymbol{\sigma}) \quad \text { is extremal in } \Omega_{M}\right\} .
$$

The homogenization problem consists in determining the set $\mathcal{A} \times \mathcal{E}$ of admissible and extremal fields. Owing to the non linearity of the problem, no exact solution can be found in general. Due to this difficulty, we present an equivalent variational formulation, more appropriate for simple approximations, thanks to relevant choice of trial fields and minimization procedure. By Hill's lemma, any admissible couple $(\boldsymbol{v}, \boldsymbol{\sigma})$ complies with:

$$
\boldsymbol{D}: \Sigma=\frac{1}{|\Omega|} \int_{\Omega} \boldsymbol{d}(\boldsymbol{v}): \boldsymbol{\sigma} d V=\frac{1}{|\Omega|} \int_{\Omega_{M}} \boldsymbol{d}(\boldsymbol{v}): \boldsymbol{\sigma} d V
$$

This suggests introducing the following two field macroscopic bifunctional:

$$
B\left(\boldsymbol{v}^{\prime}, \boldsymbol{\sigma}^{\prime}\right)=\frac{1}{|\Omega|} \int_{\Omega_{M}} b\left(\boldsymbol{d}\left(\boldsymbol{v}^{\prime}\right), \boldsymbol{\sigma}^{\prime}\right) d V-\boldsymbol{D}: \boldsymbol{\Sigma}
$$


As previously indicated, we are interested for homogenization purpose in finding the admissible and extremal couples $(\boldsymbol{v}, \boldsymbol{\sigma})$. In fact, they are solutions of the following simultaneous minimization problems:

$$
B(\boldsymbol{v}, \boldsymbol{\sigma})=\min _{\boldsymbol{v}^{\prime} \in \mathcal{K}_{a}} B\left(\boldsymbol{v}^{\prime}, \boldsymbol{\sigma}\right)=\min _{\boldsymbol{\sigma}^{\prime} \in \mathcal{S}_{a}} B\left(\boldsymbol{v}, \boldsymbol{\sigma}^{\prime}\right)=0 .
$$

Indeed, if $\left(\boldsymbol{v}^{\prime}, \boldsymbol{\sigma}^{\prime}\right)$ is admissible, relation (21) and (8) entail:

$$
B\left(\boldsymbol{v}^{\prime}, \boldsymbol{\sigma}^{\prime}\right)=\frac{1}{|\Omega|} \int_{\Omega_{M}}\left(b\left(\boldsymbol{d}\left(\boldsymbol{v}^{\prime}\right), \boldsymbol{\sigma}^{\prime}\right)-\boldsymbol{d}\left(\boldsymbol{v}^{\prime}\right): \boldsymbol{\sigma}^{\prime}\right) d V \geq 0 .
$$

In particular, this occurs for admissible couples $\left(\boldsymbol{v}^{\prime}, \boldsymbol{\sigma}\right),\left(\boldsymbol{v}, \boldsymbol{\sigma}^{\prime}\right),(\boldsymbol{v}, \boldsymbol{\sigma})$. Moreover, for the latter, owing to (9):

$$
B(\boldsymbol{v}, \boldsymbol{\sigma})=0 .
$$

In short, one has for all admissible fields $\boldsymbol{v}^{\prime} \in \mathcal{K}_{a}$ and $\boldsymbol{\sigma}^{\prime} \in \mathcal{S}_{a}$ :

$$
B\left(\boldsymbol{v}^{\prime}, \boldsymbol{\sigma}\right) \geq B(\boldsymbol{v}, \boldsymbol{\sigma})=0 \quad \text { and } \quad B\left(\boldsymbol{v}, \boldsymbol{\sigma}^{\prime}\right) \geq B(\boldsymbol{v}, \boldsymbol{\sigma})=0,
$$

which prove $(22)$.

Now, let us discuss some relevant aspects of the variational principles for a rigid perfectly plastic matrix such as the one described in the previous sections. The set of plastically admissible velocity and stress fields are respectively defined as:

$$
\begin{aligned}
& \mathcal{K}_{p}=\left\{\boldsymbol{v} \quad \text { s.t. } \quad H(\boldsymbol{d}(\boldsymbol{v})) \leq 0 \quad \text { in } \quad \Omega_{M}\right\} \\
& \mathcal{S}_{p}=\left\{\begin{array}{ll}
\boldsymbol{\sigma} & \text { s.t. }
\end{array} \quad F(\boldsymbol{\sigma}) \leq 0 \quad \text { in } \quad \Omega_{M}\right\} .
\end{aligned}
$$

while the sets of licit velocity and stress fields are respectively $\mathcal{K}_{l}=\mathcal{K}_{a} \cap \mathcal{K}_{p}$ and $\mathcal{S}_{l}=\mathcal{S}_{a} \cap \mathcal{S}_{p}$. We considered the finite valued bifunctional:

$$
B_{0}\left(\boldsymbol{v}^{\prime}, \boldsymbol{\sigma}^{\prime}\right)=\frac{1}{|\Omega|} \int_{\Omega_{M}} b_{0}\left(\boldsymbol{d}\left(\boldsymbol{v}^{\prime}\right), \boldsymbol{\sigma}^{\prime}\right) d V-\boldsymbol{D}: \boldsymbol{\Sigma} .
$$

the finite valued bipotential $b_{0}$ being introduced in (11).

Hence, the bipotential-based variational homogenization problem becomes:

$$
B_{0}(\boldsymbol{v}, \boldsymbol{\sigma})=\min _{\boldsymbol{v}^{\prime} \in \mathcal{K}_{l}} B_{0}\left(\boldsymbol{v}^{\prime}, \boldsymbol{\sigma}\right)=\min _{\boldsymbol{\sigma}^{\prime} \in \mathcal{S}_{l}} B_{0}\left(\boldsymbol{v}, \boldsymbol{\sigma}^{\prime}\right)=0 .
$$

Note that the determination of the above macroscopic bifunctional can be done by means of any of the two minimization principles, providing that the exact stress field or exact velocity field is given. 


\subsection{Application of the variational principle to the plastic porous material}

For a rigid perfectly plastic matrix, since $b_{0}$ is positively homogeneous of order one in $\boldsymbol{d}$, there is a trivial kinematical solution to the previous problem (equation (25) together with (24)) where $\boldsymbol{v}$ and $\boldsymbol{D}$ vanish. The limit analysis approach consists in finding non trivial solutions qualified as ruin mechanisms. It is expected that these non trivial solutions exist only under an equality condition on $\Sigma$ that can be interpreted as the equation of the macroscopic yielding surface in the model.

It is worth noting that if both $\boldsymbol{D}$ and $\boldsymbol{\Sigma}$ are chosen arbitrarily, there is in general no solution to the problem (25). In a practical point of view, it is more convenient for instance to fix only $\boldsymbol{\Sigma}$ and to find $\boldsymbol{D}$ and $\boldsymbol{v}$ satisfying the first minimization problem in (25). Introducing Lagrange's multiplier field $\boldsymbol{x} \mapsto \Lambda(\boldsymbol{x})$, this constrained minimization problem is transformed into an equivalent saddle-point problem

$$
\max _{\Lambda \geq 0} \min _{\boldsymbol{v} \in \mathcal{K}_{a}}\left(\mathcal{L}(\boldsymbol{v}, \boldsymbol{\sigma}, \Lambda)=B_{0}(\boldsymbol{v}, \boldsymbol{\sigma})+\frac{1}{|\Omega|} \int_{\Omega_{M}} \Lambda H(\boldsymbol{d}) d V\right) .
$$

We perform a first approximation by imposing Lagrange's multiplier field to be uniform in $\Omega_{M}$ :

$$
\max _{\Lambda \geq 0} \min _{\boldsymbol{v} \in \mathcal{K}_{a}}\left(\mathcal{L}(\boldsymbol{v}, \boldsymbol{\sigma}, \Lambda)=B_{0}(\boldsymbol{v}, \boldsymbol{\sigma})+\Lambda \frac{1}{|\Omega|} \int_{\Omega_{M}} H(\boldsymbol{d}) d V\right),
$$

that is equivalent to minimize the bifunctional $B_{0}$ under the relaxed kinematical condition:

$$
\frac{1}{|\Omega|} \int_{\Omega_{M}} H(\boldsymbol{d}) d V=0
$$

Satisfying the kinematical condition only in an average sense but not locally anywhere in $\Omega_{M}$ is a strong approximation but leading to easier calculations. As consequence of this approximation, it is crucial to remark that the minimum of $B_{0}$ may not be expected to be zero. Nevertheless, in the spirit of Ladevèze's method of the error on the constitutive law (Ladevèze , 1975; Ladevèze et al. , 1986, 1991, 1997, 2001, 2006a,b), its value for the minimizer can be used as a variational error estimator (Fortin et al., 1999). The minimum principle allows obtaining the "better" solution within the framework imposed by the approximations.

Introducing

$$
Y(\boldsymbol{v}, \boldsymbol{\sigma}, \Lambda)=\frac{1}{|\Omega|} \int_{\Omega_{M}} b_{0}(\boldsymbol{v}, \boldsymbol{\sigma}) d V+\Lambda \frac{1}{|\Omega|} \int_{\Omega_{M}} H(\boldsymbol{d}) d V
$$

and considering (24), the Lagrangian function can be recast into

$$
\mathcal{L}(\boldsymbol{v}, \boldsymbol{\sigma}, \Lambda)=Y(\boldsymbol{v}, \boldsymbol{\sigma}(\boldsymbol{\Sigma}), \Lambda)-\boldsymbol{D}: \boldsymbol{\Sigma}
$$

from which, as it will be shown by introducing the trial stress and velocity fields, one obtains the macroscopic criterion and flow rule. 
For now, let us just indicate that the ultimate step is to solve the Saddle-point problem by computing its subdifferentials with respect to parameters $\boldsymbol{D}$ :

$$
\frac{\partial \mathcal{L}}{\partial \boldsymbol{D}}(\Lambda, \Sigma)=0
$$

Eliminating the Lagrangian multiplier $\Lambda$ in the system of functionals (29), one obtains

$$
\mathcal{F}(\Sigma(\phi, \psi, f))=0
$$

A priori, the above macroscopic criterion depends not only on the porosity $f$ and the friction angle $\phi$, but also on the dilatancy angle $\psi$ of the matrix.

For completeness, the macroscopic non-associated flow rule, with the boundary conditions $\boldsymbol{v}=\boldsymbol{D} \cdot \boldsymbol{x}$, can be directly obtained from the stationnarity of the lagrangian function (28) with respect to the multiplier $\Lambda$ :

$$
\mathcal{G}=\frac{1}{|\Omega|} \int_{\Omega_{M}} H(\boldsymbol{d}) d V=0
$$

\section{The hollow sphere model with a non-associated Drucker-Prager matrix}

The major objective of this section is to apply the above bipotential-based variational approach and limit analysis technique to the hollow sphere model, which is made up of a spherical void embedded in a homothetic cell of a rigid-plastic isotropic and homogeneous matrix, the latter being described by a non-associated Drucker-Prager model. The inner and outer radii of the hollow sphere are respectively denoted $a$ and $b$, giving the void volume fraction $f=(a / b)^{3}<1$. The hollow sphere is subjected at its exterior boundary to a uniform strain rate tensor $\boldsymbol{D}$ (see Fig. 2).

Primarily, we aim at deriving a macroscopic criterion for the non-associated porous material and the corresponding flow rule.

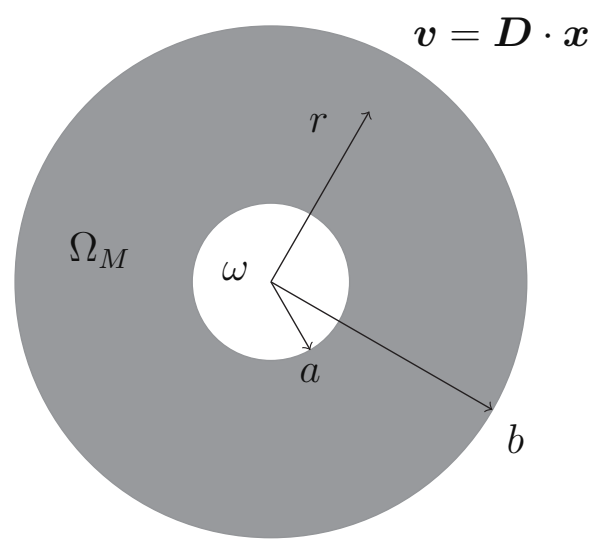

Figure 2: Hollow sphere model 


\subsection{Proposed trial stress and velocity fields}

As mentioned in Section 4, in order to derive the macroscopic model, it is indispensable to propose a couple of trial stress and velocity fields. In order to limit the errors due to approximations, we will consider trial fields for which the macroscopic model is exact at least for pure hydrostatic loadings. In Cheng et al. (2012), we obtain closed analytical formula for the limit hydrostatic stresses. For this case, the stress field and limit load do not depend on the dilatancy angle $\psi$ and they are identical to the ones of the associated case with same friction angle $\phi$, previously obtained in Thoré et al. (2009). Only the collapse mechanism is dilatancy angle dependent. This insensitivity of the hydrostatic limit load to the dilatancy angle agrees with the model of Maghous et al. (2009) already mentioned. For this reason, and taking into account the symmetry of the hollow sphere model, the trial stress field is considered as the sum of the two following fields:

- A heterogeneous part corresponding to the exact field under pure hydrostatic loadings (Thoré et al., 2009); it reads, in spherical coordinates with orthonormal frame $\left\{\mathbf{e}_{r}, \mathbf{e}_{\phi}, \mathbf{e}_{\theta}\right\}:$

$$
\boldsymbol{\sigma}^{(1)}=A_{0}\left(\frac{b}{r}\right)^{3 \gamma}\left[\boldsymbol{e}_{r} \otimes \boldsymbol{e}_{r}+\left(1-\frac{3 \gamma}{2}\right)\left(\boldsymbol{e}_{\theta} \otimes \boldsymbol{e}_{\theta}+\boldsymbol{e}_{\phi} \otimes \boldsymbol{e}_{\phi}\right)\right]
$$

where $A_{0}$ is a constant to be determined, $s=1+2 \epsilon \alpha$ and $\gamma=1-\frac{2 \epsilon \alpha}{1+2 \epsilon \alpha}$, with a loading parameter $\epsilon= \pm 1$, which will be interpreted later.

- A homogeneous part in the cylindrical coordinates with orthonormal frame $\left\{\mathbf{e}_{\rho}, \mathbf{e}_{\phi}, \mathbf{e}_{z}\right\}$ :

$$
\boldsymbol{\sigma}^{(2)}=A_{1}\left(\boldsymbol{e}_{\rho} \otimes \boldsymbol{e}_{\rho}+\boldsymbol{e}_{\phi} \otimes \boldsymbol{e}_{\phi}\right)+A_{2} \boldsymbol{e}_{z} \otimes \boldsymbol{e}_{z}
$$

where $A_{1}$ and $A_{2}$ are also constant parameters.

It should be noted that $\boldsymbol{\sigma}^{(2)}$ allows to capture the macroscopic shear effect.

The resultant three parameters based trial stress field is defined in the matrix $\Omega_{M}$ as:

$$
\sigma=\sigma^{(1)}+\sigma^{(2)}
$$

Note that a vanishing stress field is considered in the void $\omega$.

It is worth to remark that with a stress field is in internal equilibrium, one has:

$$
\Sigma^{\text {void }}=\frac{1}{|\Omega|} \int_{\omega} \boldsymbol{\sigma} d V=\frac{1}{|\Omega|} \int_{\partial \omega}(\boldsymbol{\sigma} \boldsymbol{n}) \otimes \boldsymbol{x} d S
$$

Hence:

$$
\Sigma_{m}^{v o i d}=\frac{1}{|\Omega|} \int_{\omega} \sigma_{m} d V=(3 V)^{-1} \int_{\partial \omega} \boldsymbol{x} \cdot(\boldsymbol{\sigma} \boldsymbol{n}) d S
$$

As the continuity condition:

$$
(\boldsymbol{\sigma} \boldsymbol{n})_{-}+(\boldsymbol{\sigma} \boldsymbol{n})_{+}=0 \text { on } \partial \omega
$$


is difficult to satisfy by the very simple chosen trial field, we relax it as follows:

$$
(3 V)^{-1} \int_{\partial \omega} \boldsymbol{x} \cdot\left((\boldsymbol{\sigma} \boldsymbol{n})_{-}+(\boldsymbol{\sigma} \boldsymbol{n})_{+}\right) d S=0
$$

which can be equivalently written as:

$$
f^{-\gamma} A_{0}+\frac{2 A_{1}+A_{2}}{3}=0
$$

On the other hand, the macroscopic stress field is:

$$
\boldsymbol{\Sigma}=A_{0}\left(1-f^{1-\gamma}\right) \mathbf{1}+(1-f)\left[A_{1}\left(\boldsymbol{e}_{x} \otimes \boldsymbol{e}_{x}+\boldsymbol{e}_{y} \otimes \boldsymbol{e}_{y}\right)+A_{2} \boldsymbol{e}_{z} \otimes \boldsymbol{e}_{z}\right],
$$

Taking into account (37), the macroscopic mean stress is:

$$
\Sigma_{m}=A_{0}\left(1-f^{-\gamma}\right),
$$

while the macroscopic deviatoric stress reads:

$$
\Sigma_{e}=(1-f)\left|A_{1}-A_{2}\right| \text {. }
$$

These two last relations allow to express the stress parameters in terms of the macroscopic stress:

$$
A_{0}=\frac{\Sigma_{m}}{1-f^{-\gamma}}, \quad\left|A_{1}-A_{2}\right|=\frac{\Sigma_{e}}{1-f} .
$$

Next, following Guo et al. (2008) (see also (Thoré et al., 2009)), we adopt, in cylindrical coordinates, the following trial velocity field which depend on the dilatancy angle $\psi$, not on the friction angle $\phi$,

$$
\boldsymbol{v}=C_{0}\left(\frac{b}{r}\right)^{3 / \tilde{s}}\left(\rho \boldsymbol{e}_{\rho}+z \boldsymbol{e}_{z}\right)+C_{1} \rho \boldsymbol{e}_{\rho}+C_{2} z \boldsymbol{e}_{z},
$$

with $r=\sqrt{\rho^{2}+z^{2}}, \tilde{s}=1+2 \epsilon \beta$, where $\epsilon$ is the sign of $C_{0}$. The first term is the exact solution for the hydrostatic case (Cheng et al., 2012). As in Gurson's model (Gurson, 1977) and in its extension to pressure sensitive dilatant materials (Guo et al., 2008), this term is completed by two linear terms in order to capture the shear effects.

$\boldsymbol{D}$ being the applied macroscopic strain rate, the trial velocity field (42) must comply with the boundary conditions:

$$
\boldsymbol{v}=\boldsymbol{D} \cdot \boldsymbol{x}
$$

In the case of axisymmetric macroscopic strain rate $\left(\boldsymbol{D}=D_{x x}\left(\boldsymbol{e}_{x} \otimes \boldsymbol{e}_{x}+\boldsymbol{e}_{y} \otimes \boldsymbol{e}_{y}\right)+\right.$ $\left.D_{z z} \boldsymbol{e}_{z} \otimes \boldsymbol{e}_{z}\right)$, considered in the present study, $C_{0}, C_{1}$ and $C_{2}$ are such that:

$$
\begin{aligned}
& D_{m}=\frac{1}{3} \operatorname{tr} \boldsymbol{D}=C_{0}+\frac{1}{3}\left(2 C_{1}+C_{2}\right) \\
& D_{z z}-D_{x x}=\frac{2}{3}\left(C_{1}-C_{2}\right)
\end{aligned}
$$

from which it follows:

$$
D_{e}=\sqrt{\frac{2}{3} \boldsymbol{D}^{\prime}: \boldsymbol{D}^{\prime}}=\frac{2}{3}\left|C_{1}-C_{2}\right|
$$

$\boldsymbol{D}^{\prime}$ being the deviatoric part of $\boldsymbol{D}$. 


\subsection{Closed-form expression of the macroscopic bifunctional}

In order to derive the non-associated macroscopic model by solving the saddle point problem (26), we aim now at parametrically expressing the macroscopic bifunctional (24) thanks to the proposed trial stress (34) and trial velocity fields (42). It should be pay attention that the bipotential (14) depends on the microscopic mean stress $\sigma_{m}$, mean strain rate $d_{m}$ and equivalent strain rate $d_{e q}$. From (34) and (42), and considering (41), these quantities can be respectively calculated as

$$
\begin{gathered}
\sigma_{m}(r)=\frac{\Sigma_{m}}{1-f^{\gamma}}\left[1-\frac{f^{\gamma}}{s}\left(\frac{b}{r}\right)^{3 \gamma}\right] \\
d_{m}(r)=\frac{1}{3} \operatorname{tr} \boldsymbol{d}=\left(1-\frac{1}{\tilde{s}}\right) C_{0}\left(\frac{b}{r}\right)^{3 / \tilde{s}}+\frac{1}{3}\left(2 C_{1}+C_{2}\right) \\
d_{e q}(r)=\frac{2}{3} \sqrt{\left(C_{1}-C_{2}\right)^{2}+\left(C_{1}-C_{2}\right) \frac{3 C_{0}}{\tilde{s}}\left(\frac{b}{r}\right)^{3 / \tilde{s}}\left(3 \cos ^{2} \theta-1\right)+\left(\frac{3 C_{0}}{\tilde{s}}\right)^{2}\left(\frac{b}{r}\right)^{6 / \tilde{s}}}
\end{gathered}
$$

for which one must have in mind the relations:

$$
\begin{gathered}
d_{m}(r)=D_{m}+C_{0}\left[\left(1-\frac{1}{\tilde{s}}\right)\left(\frac{b}{r}\right)^{3 / \tilde{s}}-1\right] \\
d_{e q}(r)=\frac{2}{3} \sqrt{D_{e}^{2}+\operatorname{sign}\left(C_{1}-C_{2}\right) D_{e} \frac{3 C_{0}}{\tilde{s}}\left(\frac{b}{r}\right)^{3 / \tilde{s}}\left(3 \cos ^{2} \theta-1\right)+\left(\frac{3 C_{0}}{\tilde{s}}\right)^{2}\left(\frac{b}{r}\right)^{6 / \tilde{s}}}
\end{gathered}
$$

For simplicity, let us introduce the normalized stress tensor

$$
\boldsymbol{T}=\frac{\Sigma}{\sigma_{0}}
$$

and the contribution of the void to average strain rate

$$
\boldsymbol{D}(a)=C_{0} f^{\tilde{\gamma}} \mathbf{1}+f\left[C_{1}\left(\boldsymbol{e}_{x} \otimes \boldsymbol{e}_{x}+\boldsymbol{e}_{y} \otimes \boldsymbol{e}_{y}\right)+C_{2} \boldsymbol{e}_{z} \otimes \boldsymbol{e}_{z}\right],
$$

with $\tilde{\gamma}=1-\tilde{s}^{-1}$.

Combining (14), (22) and (50), the normalized macroscopic bifunctional can be obtained as follows:

$$
\bar{B}_{0}(\boldsymbol{v}, \boldsymbol{\sigma})=\frac{B_{0}(\boldsymbol{v}, \boldsymbol{\sigma})}{\sigma_{0}}=\frac{1}{3 \alpha} \operatorname{tr}(\boldsymbol{D}-\boldsymbol{D}(a))+\left(1-\frac{\beta}{\alpha}\right) \hat{\Pi}(\boldsymbol{v}, \boldsymbol{\sigma})-\boldsymbol{D}: \boldsymbol{T}
$$

with

$$
\hat{\Pi}(\boldsymbol{v}, \boldsymbol{\sigma})=\frac{1}{|\Omega|} \int_{\Omega_{M}} d_{e q} d V-\frac{1}{|\Omega|} \int_{\Omega_{M}} 3 \alpha \frac{\sigma_{m}}{\sigma_{0}} d_{e q} d V
$$

Hence, it is convenient to introduce

$$
\Pi(\boldsymbol{v})=\frac{1}{|\Omega|} \int_{\Omega_{M}} d_{e q} d V,
$$


which by considering (47) and (43), can be reduced into

$$
\Pi\left(C_{0}, D_{e}\right)=D_{e} \int_{f}^{1} K(\xi) \sqrt{1+\tau^{2} x^{-2 / \tilde{s}}} d x,
$$

with

$$
\begin{gathered}
\tau=\frac{2 C_{0}}{\tilde{s} D_{e}}, \quad x=\left(\frac{r}{b}\right)^{3}, \quad \xi=\frac{2 \tau x^{-1 / \tilde{s}}}{1+\tau^{2} x^{-2 / \tilde{s}}} \operatorname{sign}\left(C_{1}-C_{2}\right), \quad|\xi| \leq 1 . \\
K(\xi)=\frac{1}{2} \int_{0}^{\pi} \sqrt{1+\frac{1}{2}\left(3 \cos ^{2} \theta-1\right) \xi} \sin \theta d \theta
\end{gathered}
$$

Putting (45) into (53), $\hat{\Pi}$ can be recast into the following parametric form

$$
\hat{\Pi}\left(C_{0}, D_{e}, T_{m}\right)=\left(1-\frac{3 \alpha T_{m}}{1-f^{\gamma}}\right) \Pi\left(C_{0}, D_{e}\right)+\frac{3 \alpha T_{m}}{1-f^{\gamma}} \frac{f^{\gamma}}{s} I\left(D_{e}\right)
$$

with

$$
I\left(D_{e}\right)=D_{e} \int_{f}^{1} x^{-\gamma} K(\xi) \sqrt{1+\tau^{2} x^{-2 / \tilde{s}}} d x .
$$

Finally, the closed form expression of the macroscopic bifunctional reads:

$$
\begin{aligned}
\bar{B}_{0}\left(C_{0}, D_{m}, D_{e}, T_{m}, T_{e}\right)= & \frac{1}{\alpha}\left[(1-f) D_{m}-\left(f^{\tilde{\gamma}}-f\right) C_{0}\right] \\
& +\left(1-\frac{\beta}{\alpha}\right) \hat{\Pi}\left(C_{0}, D_{e}, T_{m}\right)-\left(D_{e} T_{e}+3 D_{m} T_{m}\right)
\end{aligned}
$$

This constitute one of the key practical respect of the study.

\subsection{Determination of the macroscopic criterion and of the macroscopic flow rule}

Having in hand the bifunctional $B_{0}$, we are already now to determine the kinematical admissibility condition (27), written as

$$
\beta \Pi\left(C_{0}, D_{e}\right)-\left[(1-f) D_{m}-\left(f^{\tilde{\gamma}}-f\right) C_{0}\right]=0
$$

which plays the role of the macroscopic flow rule.

Concerning the determination of the macroscopic criterion, let us first introduce a normalized multiplier $\bar{\Lambda}=\Lambda / \sigma_{0}$, the normalized lagrangian can be written as

$$
\begin{aligned}
\overline{\mathcal{L}}\left(C_{0}, D_{m}, D_{e}, T_{m}, T_{e}, \bar{\Lambda}\right)= & \frac{\mathcal{L}\left(C_{0}, D_{m}, D_{e}, T_{m}, \Lambda\right)}{\sigma_{0}} \\
= & \left(\frac{1}{\alpha}-\bar{\Lambda}\right)\left[(1-f) D_{m}-\left(f^{\tilde{\gamma}}-f\right)\right]+\bar{\Lambda} \beta \Pi\left(C_{0}, D_{e}\right) \\
& +\left(1-\frac{\beta}{\alpha}\right) \hat{\Pi}\left(C_{0}, D_{e}, T_{m}\right)-\left(D_{e} T_{e}+3 D_{m} T_{m}\right)
\end{aligned}
$$


For the minimization of the macroscopic bifunctional (59), one needs to calculate the partial derivatives of the normalized lagrangian (61). Considering

$$
\tau=\tau\left(C_{0}, D_{e}\right), \quad \Pi\left(C_{0}, D_{e}\right)=\Pi\left(\tau, D_{e}\right), \quad \hat{\Pi}\left(C_{0}, D_{e}, T_{m}\right)=\Pi\left(\tau, D_{e}, T_{m}\right)
$$

its stationarity with respect to $D_{e}, D_{m}$ and $C_{0}$ gives for the normalized stress tensor $\boldsymbol{T}=\frac{\boldsymbol{\Sigma}}{\sigma_{0}}$ :

$$
\begin{gathered}
T_{e}(\tau)=\bar{\Lambda} \beta \Pi_{,_{e}}(\tau)+\left(1-\frac{\beta}{\alpha}\right) \hat{\Pi}_{D_{e}}(\tau) \\
3 T_{m}(\tau)=\left(\frac{1}{\alpha}-\bar{\Lambda}(\tau)\right)(1-f), \\
\bar{\Lambda} \beta \Pi_{,_{0}}(\tau)+\left(1-\frac{\beta}{\alpha}\right) \hat{\Pi}_{C_{0}}(\tau)-\left(\frac{1}{\alpha}-\bar{\Lambda}(\tau)\right)\left(f^{\tilde{\gamma}}-f\right)=0 .
\end{gathered}
$$

from which, we deduce the expression of the normalized multiplier:

$$
\bar{\Lambda}(\tau)=\frac{\frac{1}{\alpha}\left(f^{\tilde{\gamma}}-f\right)+\left(\frac{\beta}{\alpha}-1\right) \hat{\Pi}_{C_{0}}(\tau)}{f^{\tilde{\gamma}}-f+\beta \Pi_{C_{0}}(\tau)} .
$$

Eliminating $\bar{\Lambda}$ in (62) and (63), delivers the closed-form macroscopic criterion in the form:

$$
\begin{aligned}
& T_{e}=\frac{(f \tilde{\gamma}-f)\left[\frac{\beta}{\alpha} \Pi_{D_{e}}+\left(1-\frac{\beta}{\alpha}\right) \hat{\Pi}_{D_{e}}\right]+\left(1-\frac{\beta}{\alpha}\right) \beta\left(\Pi_{C_{0}} \hat{\Pi}_{, D_{e}}-\Pi_{, D_{e}} \hat{\Pi}_{C_{0}}\right)}{f \tilde{\gamma}-f+\beta \Pi_{C_{0}}} \\
& 3 T_{m}=(1-f) \frac{\frac{\beta}{\alpha} \Pi_{C_{0}}+\left(1-\frac{\beta}{\alpha}\right) \hat{\Pi}_{C_{0}}}{f \tilde{\gamma}-f+\beta \Pi_{C_{0}}} .
\end{aligned}
$$

in which $\hat{\Pi}$ is given by (57), and it is recalled that $\tilde{\gamma}=\frac{2 \epsilon \beta}{1+2 \epsilon \beta}$.

The explicit expressions for $\Pi_{, C_{0}}, \Pi_{, D_{e}}, \hat{\Pi}_{C_{0}}, \hat{\Pi}_{, D_{e}}$ and their antiderivatives $\Pi, \hat{\Pi}$ are calculated and detailed in Appendix A. It is worthy to noted that the above macroscopic criterion (65) is established in a parametric form which depends on the strain rate ratio $\tau$ (55) corresponding to the velocity imposed condtion $\boldsymbol{v}=\boldsymbol{D} \cdot \boldsymbol{x}$. More precisely, the points of plastic limit stress curve can be obtained from the parametric macroscopic criterion (65) for different fixed values of $\tau$. This also allows to deduce the normalized triaxiality $\mathcal{T}=T_{m} / T_{e}$.

Finally, the stationarity of the normalized lagrangian (61) with respect to $\bar{\Lambda}$ gives directly the macroscopic flow rule (see (60)).

Note also that, macroscopic associated flow rule is obtained by $\beta=\alpha$, non-associated one otherwise. Let us recall from (55) that

$$
C_{0}=\frac{\tilde{s}}{2} \tau D_{e}
$$

and introduce

$$
\mathcal{P}=\frac{\Pi}{D_{e}}
$$


which only depends on $\tau$. Inserting (66) and (67) into (60) provides a new form of the admissibility condition (60) (flow rule):

$$
\frac{D_{m}}{D_{e}}=\frac{1}{1-f}\left[\beta \mathcal{P}(\tau)+\left(f^{\tilde{\gamma}}-f\right) \frac{\tilde{s}}{2} \tau\right]
$$

Hence, the plastic flow direction $\Upsilon$ can be obtained once the value of $\tau$ is pre-proposed,

$$
\Upsilon=\operatorname{acot}\left[\frac{1}{1-f}\left(\beta \mathcal{P}(\tau)+\left(f^{\tilde{\gamma}}-f\right) \frac{\tilde{s}}{2} \tau\right)\right]
$$

Finally, owing to the matrix compressibility, the void growth rate readily reads,

$$
\dot{f}=3(1-f) D_{m}-\frac{1}{|\Omega|} \int_{\Omega_{M}} \operatorname{tr} \boldsymbol{d} d V
$$

which, by considering (42), (46) and (66), takes the final expression,

$$
\dot{f}=3\left(f^{\tilde{\gamma}}-f\right) C_{0}=\frac{3}{2}\left(f^{\tilde{\gamma}}-f\right) \tilde{s} \tau D_{e}
$$

Note that (65), (68) and (71) are probably some of the most important and practical results of the study. They are the basic blocks of the non-associated constitutive law of the porous material having a non associated Drucker-Prager matrix.

\section{Examination of some special cases}

We analyze in this subsection the predictions obtained for some special cases for which results are available in literature. Let us first note that the general problem involves three constants defining the velocity field (42). These constants are linked by the three relations (43), (44) and (60). These equations can be easily explicit in the particular cases examined below.

- Hydrostatic case: $D_{e}=0$ and $C_{0} \neq 0$

From (43) and (47), the microscopic equivalent strain rate in this case reads

$$
d_{e q}(r)=\frac{2\left|C_{0}\right|}{\tilde{s}}\left(\frac{b}{r}\right)^{3 / \tilde{s}}
$$

It follows from (54) and (58) that

$$
\Pi=\frac{C_{0}}{\beta}\left(1-f^{\tilde{\gamma}}\right), \quad I=\frac{2\left|C_{0}\right|}{\tilde{s}} \frac{1-f^{\tilde{\gamma}-\gamma}}{\tilde{\gamma}-\gamma}
$$

in which $\tau=\frac{C_{0}}{D_{e}}$ has been also considered. Hence, taking also into account (57), the macroscopic stress is given from (65) in the form:

$$
T_{e}=\frac{\Sigma_{e}}{\sigma_{0}}=0, \quad T_{m}=\frac{\Sigma_{m}}{\sigma_{0}}=\frac{1}{3 \alpha}\left(1-f^{\gamma}\right),
$$


which is the plastic limit state for the pure hydrostatic loading $\Sigma_{e}=0$ (traction and compression). This result corresponds to the exact solution in non-associated case derived by Cheng et al. (2012), which have also verified that for the hydrostatically loaded hollow sphere, the limit loads for the non-associated case are the same as for the corresponding associated one given by Guo et al. (2008) (see also (Thoré et al., 2009)). Finally, due to $D_{e}=0,(60)$ readily implies $C_{0}=D_{m}$ and then, by (71), $\dot{f}=3\left(f^{\tilde{\gamma}}-f\right) D_{m}$.

- $C_{0}=0$ and $D_{e} \neq 0$

In this case, from (47), (54) and (58) we have

$$
\begin{gathered}
d_{e q}=\frac{2}{3}\left|C_{1}-C_{2}\right|=\frac{2}{3} D_{e} \\
\Pi=D_{e}(1-f), \quad I=D_{e} \frac{1-f^{1-\gamma}}{1-\gamma}
\end{gathered}
$$

The macroscopic limit stresses can be obtained from (65)

$$
T_{m}=\frac{\Sigma_{m}}{\sigma_{0}}=0, \quad T_{e}=\frac{\Sigma_{e}}{\sigma_{0}}=1-f
$$

Similarly, the plastic flow direction can be derived from (68) or (69):

$$
\frac{D_{m}}{D_{e}}=\beta, \quad \text { or } \quad \Upsilon=\operatorname{acot} \beta
$$

In this case, the macroscopic admissibility condition appears as the exact couterpart of the microscopic one. For completeness to this case, the void growth rate can be immediately obtained from (71), that is $\dot{f}=0$. All of the above results obtained from $C_{0}=0$ reflects and proves that the hollow sphere model is under a pure shear loading (73). They provide the same result as the solution obtained by Gurson (1977) and Guo et al. (2008). Obviously, it leads to the conclusion that no matter the matrix of the porous media take a normality rule or not, solution for pure shear loading depends only on the value of porosity, neither on the friction angle nor the dilatancy one. This is a limitation which comes from the simplicity of the trial fields. Equally important, the plastic flow follows a constant and regularly direction (74), which is independent on the friction angle $\phi$. It can be calculated when and only when the dilatancy angle $\psi$ is fixed.

- Case of associated matrix

When the matrix complies with an associated flow rule, $\psi=\phi$ and $\beta=\alpha$ in Eqs. (1) and (2). It is worthy to indicate that for the pressure-sensitive matrix there is such as $\beta=\alpha \neq 0$. Consequently, one gets from (65) the following macroscopic criterion

$$
\begin{aligned}
& T_{e}=\frac{f^{\gamma}-f}{f^{\gamma}-f+\alpha \frac{\partial \Pi}{\partial C_{0}}} \frac{\partial \Pi}{\partial D_{e}} \\
& 3 T_{m}=\frac{1-f}{f^{\gamma}-f+\alpha \frac{\partial \Pi}{\partial C_{0}}} \frac{\partial \Pi}{\partial C_{0}}
\end{aligned}
$$


and from (69) and (71) that the plastic flow direction and the void growth rate, respectively

$$
\begin{gathered}
\Upsilon=\operatorname{acot}\left[\frac{1}{1-f}\left(\alpha \mathcal{P}(\tau)+\left(f^{\gamma}-f\right) \frac{s}{2} \tau\right)\right] \\
\dot{f}=3\left(f^{\gamma}-f\right) C_{0}=\frac{3}{2}\left(f^{\gamma}-f\right) s \tau D_{e}
\end{gathered}
$$

which is precisely the so called Upper Bound Model (UBM) ${ }^{3}$ proposed in Guo et al. (2008). For completeness, we present in Appendix B the derivation of our methodology in the case of associated matrix.

- von Mises matrix

For the porous material with an incompressible matrix, for instance the von Mises yield criterion at microscopic level, the pressure-sensitive parameter $\phi$ and $\psi$ both vanish, or in another word $\alpha=\beta=0$. In this case, we have

$$
\tilde{s}=s=1, \quad \tilde{\gamma}=\gamma=0
$$

and owing to (60), one obtains $D_{m}=C_{0}$. As a result, (47) then takes the form

$$
d_{e q}=\frac{2}{3} \sqrt{D_{e}^{2}+3 D_{m} D_{e}\left(3 \cos ^{2} \theta\right)\left(\frac{b}{r}\right)^{3}+\left(3 D_{m}\right)^{2}\left(\frac{b}{r}\right)^{6}}
$$

and (65) reduced to

$$
\begin{aligned}
& T_{e}=\frac{\Sigma_{e}}{\sigma_{0}}=\frac{\partial \Pi}{\partial D_{e}} \\
& 3 T_{m}=3 \frac{\Sigma_{m}}{\sigma_{0}}=\frac{\partial \Pi}{\partial D_{m}}
\end{aligned}
$$

Considering (54) and (71), we get the following macroscopic criterion

$$
T_{e}^{2}+2 f \cosh \left(\frac{3}{2} T_{m}\right)-\left(1+f^{2}\right)=0,
$$

and the void growth equation

$$
\dot{f}=3(1-f) D_{m}
$$

which are the well-known results of Gurson (1977) obtained by a kinematical limit analysis approach.

\footnotetext{
${ }^{3}$ It should be emphasized that the macroscopic model proposed by Guo et al. (2008) cannot be seen as an upper bound. For the corresponding demonstration, readers can be referred to Cheng (2013).
} 


\section{Illustration and numerical validation of the established criterion}

In this section, the predictions of established macroscopic criterion (65) in non-associated cases will be firstly compared with the associated one (75) (see also the so called UBM of Guo et al. (2008)) in subsection 7.1. The expected influence of the non-associated feature is clearly illustrated. Next, Finite Element Method (FEM) based limit analysis computations are performed in subsection 7.2 and their results allow to assess the obtained theoretical criterion. For completeness, due to the fact that the plastic flow rule has been formulated in an implicit form except for the pure shear loading, we will perform in subsection 7.3 the illustration of the analytical plastic flow direction (68) for such a particular case, which will be validated from the corresponding FEM solutions.

\subsection{Preliminary illustration of the established criterion}

We aim now at illustrating the macroscopic criterion (65) established in subsection 5.3 both in associated and non-associated cases. As mentioned before, the matrix pressure sensitivity is characterized by the friction angle $\phi$ and the dilatancy one $\psi$ for the Drucker-Prager model (see Eqs.(1) and (2)). These two angles must satisfy the condition $0 \leq \psi \leq \phi<56^{\circ} 18^{\prime}$ (see also Eq.(5)). It is convenient to note that porosity values in geomaterials are relatively bigger comparatively to porous metals or polymers, etc.. Accordingly, in this subsection, we provide illustration of the established criterion for a porosity $f=0.2$ and friction angle $\phi=30^{\circ}$. The corresponding associated case $\psi=\phi=30^{\circ}$ is denoted AC, while two nonassociated cases are considered; they are respectively defined by dilatancy angles $\psi=15^{\circ}$ (denoted $\mathrm{NAC} 1)$ and $\psi=5^{\circ}(\mathrm{NAC} 2)$.

As already mentioned, for hydrostatic loadings (traction and compression), the non-associated cases provide the same predictions as that of the associated one (see Guo et al. (2008); Thoré et al. (2009). Note again that this observation is in full agreement with the theoretical and numerical results already established in Cheng et al. (2012). Furthermore, unlike the previous work of Maghous et al. (2009), as mentioned in sections 4 and 5.3, the non-associated cases show in general different yield loci with respect to the associated one: as expected, the yield surface for a non associated case is lower than for the associated one. Note that a decrease of the dilatancy angle leads to a weaker strength, the difference between the cases $\psi=15^{\circ}$ and $\psi=5^{\circ}$ being slight. These results will be investigated in subsection 7.2 by means of numerical results.

To simplify the presentation here, additional results are provided in Appendix C.1 (see Figs. C.10 and C.11); they allow to illustrate the effects of porosity ( $f=0.15, f=0.25$ ) on the macroscopic yield surfaces in the context of the non-associated plastic matrix. In the same appendix, effects of the friction angle $\phi$ are also provided for a porosity $f=0.2$. 


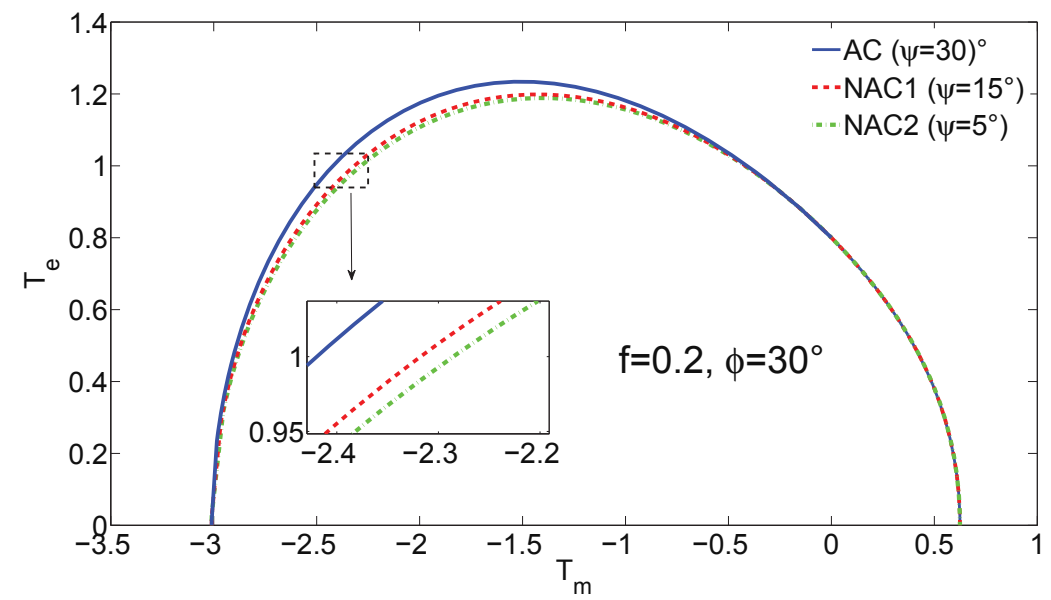

Figure 3: Comparison of yield surfaces between the associated case (denoted AC) with dilatancy angle $\psi=30^{\circ}$ and two non-associated cases (65) (denoted NAC1 and NAC2) with $\psi=15^{\circ}$ and $5^{\circ}$, respectively. Porosity: $f=0.2$; friction angle $\phi=30^{\circ}$

7.2. Numerical investigations of the macroscopic yield surface and plastic flow rule in the context of the Druker-Prager non associated matrix

In this subsection, the predictions of the established macroscopic criterion will be compared with the Finite Element Method (FEM) solutions. For the FEM analysis, we consider an axisymmetric model of the spherical shell. Hence, owing to the geometrical symmetry, only a quarter of this model is considered by adopting 1500 quadratic axisymmetric elements (see Fig.4). Moreover, the numerical analysis is carried out in the context of non-associated elastoplasticity and small deformations. The computations are performed by means of ABAQUS/Standard software and a user subroutine MPC (Multi-Points Constraints). The main reason for which we need to enforce MPC conditions in the code is that we have to impose the velocity field $\boldsymbol{v}$ from $\boldsymbol{v}=\boldsymbol{D} \cdot \boldsymbol{x}$ (on the external boundary of the hollow sphere) such that the constraint of constant macroscopic stress triaxiality $\left(T=\Sigma_{m} / \Sigma_{e}\right)$ be fulfilled. In practice, as in Guo et al. (2008), this is done by applying a constant macroscopic stress ratio $\Sigma_{\rho} / \Sigma_{z}$ corresponding to the desired $\Sigma_{m} / \Sigma_{e}$. Note that the implementation of this procedure has been already described by Cheng and Guo (2007) for their study of voids interaction and coalescence in an associated Drucker-Prager matrix. 


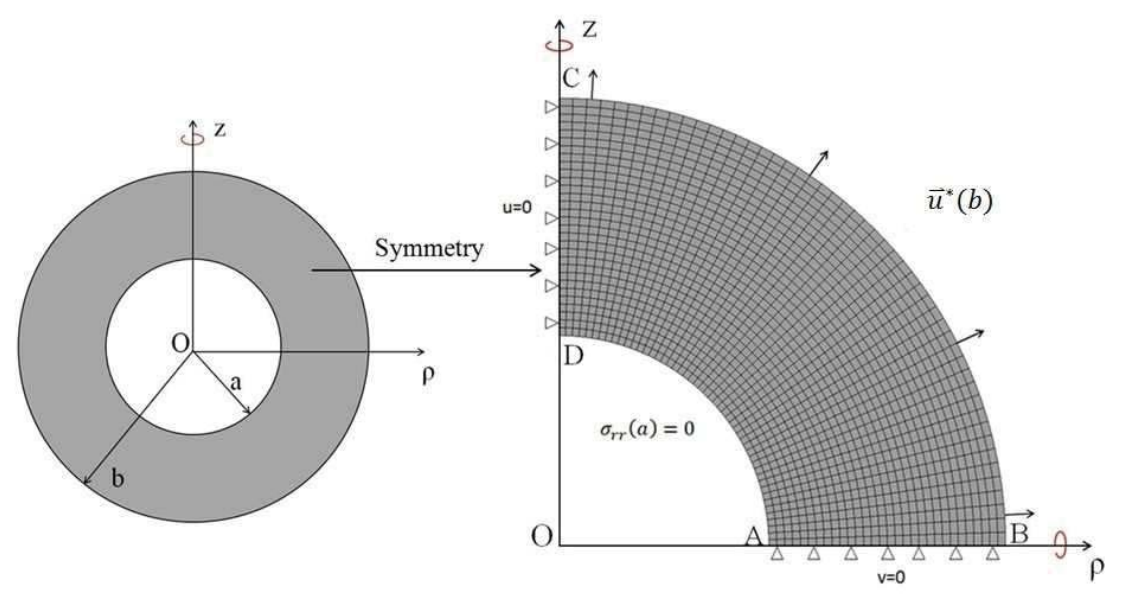

Figure 4: Hollow sphere model: Geometry of the elementary cell and boundary conditions.

Fig. 5 displays the FEM results not only for the macroscopic limit stress, but also for the direction of plastic flow. As in subsection 7.1, the values of porosity $f=0.2$ and friction angle $\phi=30^{\circ}$ are considered here. Also, the direction of plastic flow for the associated case and two non-associated cases are denoted $\operatorname{DA}\left(\psi=30^{\circ}\right)$, DNA1 $\left(\psi=15^{\circ}\right)$ and DNA2 $\left(\psi=5^{\circ}\right)$, respectively. Moreover, the numerical yield surfaces, obtained by connecting each FEM point of plastic limit state, are indicated by $\operatorname{SFA}\left(\psi=30^{\circ}\right), \operatorname{SFNA} 1\left(\psi=15^{\circ}\right)$ and SFNA2 $\left(\psi=5^{\circ}\right)$, respectively. Note that each FEM point has been obtained by performing computation at fixed stress triaxialities $\Sigma_{m} / \Sigma_{e}$ (equivalently at fixed $T_{m} / T_{e}$ ).

Coming now to the results, an excellent agreement between the DA and SFA is noted (see Fig.5), the plastic flow direction (DA) at each FEM point being normal to the yield surface (SFA). Concerning the plastic flow direction (DNA1 and DNA2) for the cases of non-associated matrix, a lack of normality to the corresponding yield surfaces (SFNA1 and SFNA2) is noted. These FEM results proves the non-associated character of the macroscopic flow rule in the case of a non-associated matrix. It must be noted that the lack of normality is more pronounced when the dilatancy angle $\psi$ is small, that is a material with a pronounced non associated matrix.

For completeness, additional results and validations are provided on Figs. 12(a), 13(a), 14(a) and 15(a) in Appendix C.2. This allows to illustrate the effects of the porosity and friction angle. 


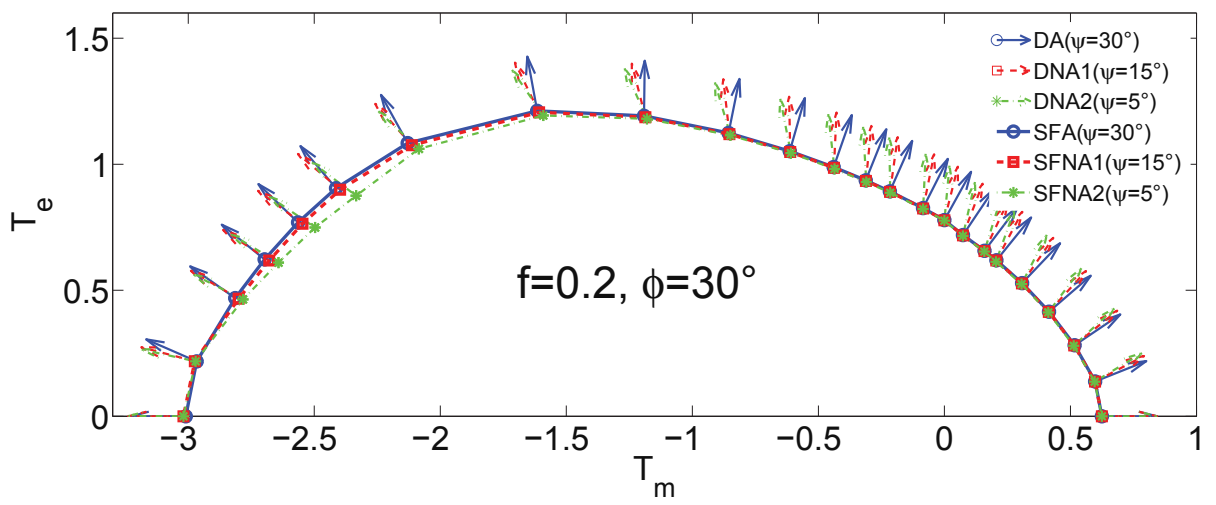

Figure 5: Illustration of FEM results: plastic flow directions (denoted DA for associated case, DNA1 and DNA2 for non-associated ones) and yield surface (denoted SFA for associated case, SFNA1 and SFNA2 for non-associated ones).

\subsection{Validations of the established criterion and of the corresponding flow rule}

For validation purpose, the analytical yield surfaces for the associated case (AC) as well as the non-associated ones (NAC1 and NAC2) are compared with the corresponding numerical limit stresses on Fig. 6. The FEM results confirm that the limit stresses of non-associated cases (denoted FNAC1 and FNAC2) and the associated one (denote FAC) are very close in the vicinity of traction dominant region $T_{m}>0$ ( or $\Sigma_{m}>0$ ). In contrary, a slight difference is observed in a part of the compression dominate region $T_{m}<0$ (or $\Sigma_{m}<0$ ). The above numerical results validate the predictive capabilities of the analytical criterion.

Let us recall that other validating results showing also the influences of the porosity and friction angle are provided on Fig. 12(b), 13(b), 14(b) and 15(b) in Appendix C.2. 


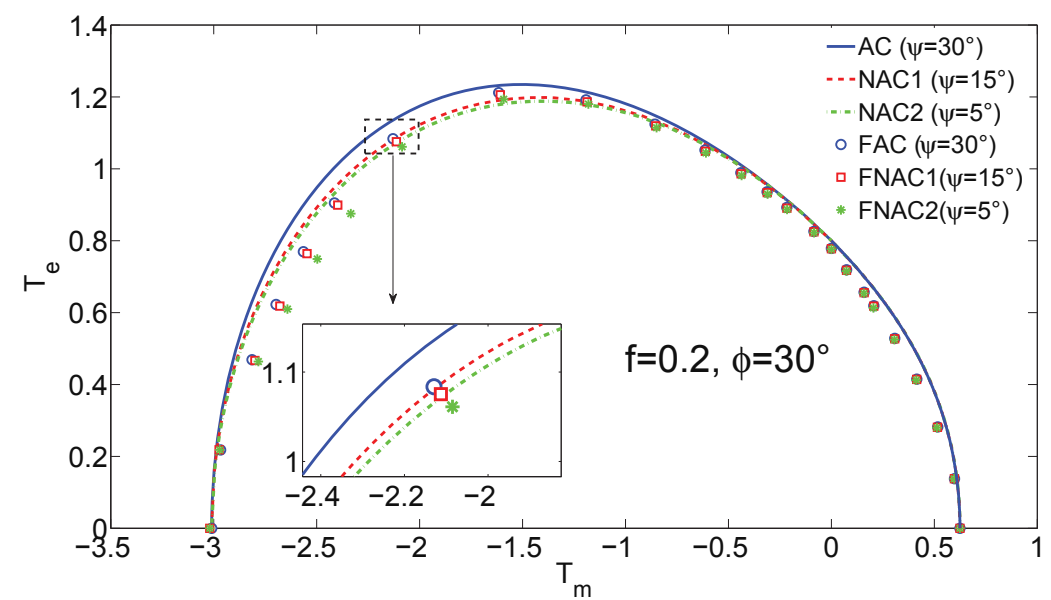

Figure 6: Comparison between the yield surfaces obtained from the established criteria (65) and FEM results (denoted FAC for associated case, FAC1 and FAC2 for non-associated ones).

Concerning the assessment of the obtained macroscopic flow rule (see Eq. (68)). We display on Fig.7 the plastic direction obtained from the analytical function (69) and that numerically computed from the FEM computation. Both of these results are illustrated with respect to the different values of macroscopic stress triaxiality $T_{m} / T_{e}$. The illustrations have been realized for $f=0.2$ and $\phi=30^{\circ}, \psi=30^{\circ}, \psi=15^{\circ}$ and $\psi=5^{\circ}$. Noticeable difference between the associated case and the non associated ones is obtained, both for the analytical results and for the numerical data. Moreover, for any fixed value of triaxiality, the obtained value of $\operatorname{acot}\left(D_{m} / D_{e}\right)$ representing the plastic flow direction is smaller when the dilatancy angle $\psi$ diminishes. Finally, very good qualitative agreement is observed between the theoretical predictions and the corresponding numerical data. 


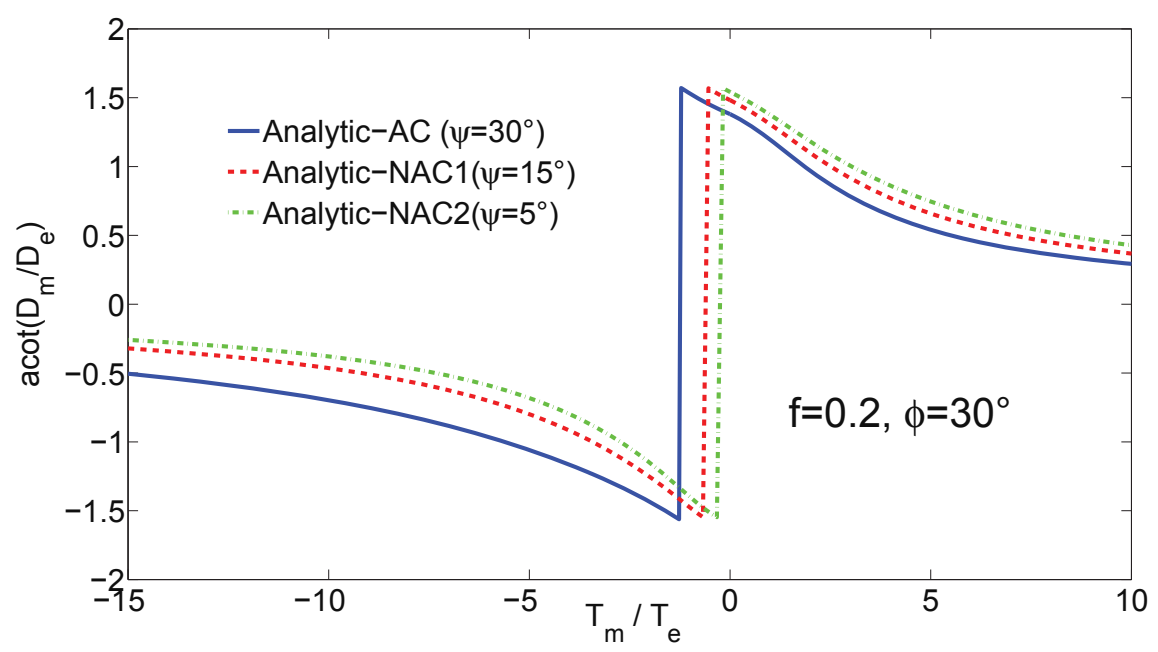

(a) Analytic

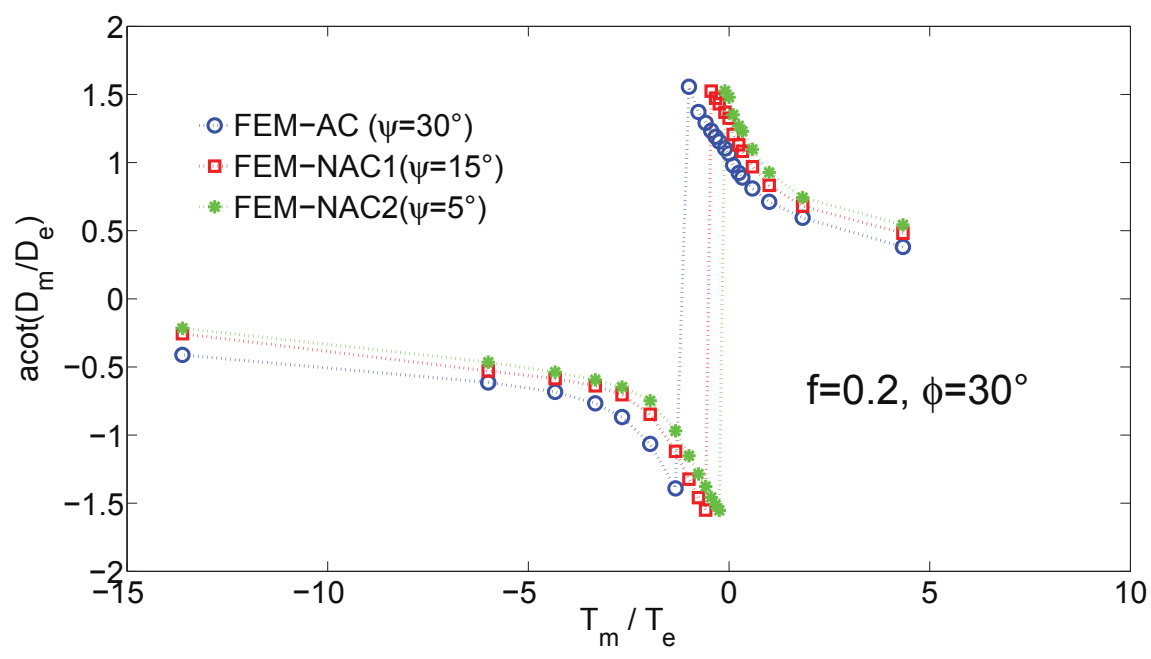

(b) FEM

Figure 7: Analytical result and FEM solutions for plastic flow direction with the fixed values of porosity $f=0.2$ and friction angle $\phi=30^{\circ}$.

At the difference of porous media with an incompressible plastic matrix (Gurson, 1977), void growth (see Eq. (71)) occurs under macroscopic pure shear loading in the case of a pressure-sensitive matrix (as considered in the present work). For this particular shear loading, Eq.(74) indicates that the plastic flow is only influenced by the dilatancy parameter $\beta$, but not by the friction one $\alpha$. Still for the pure shear, we perform on Fig. 8 a comparison of the plastic flow direction given by the analytical solution (74) and the FEM computations. The following values of fixed material parameters, $f=0.2$ and $\phi=30^{\circ}$, are considered. An excellent agreement between the analytic and FEM results is obtained. 


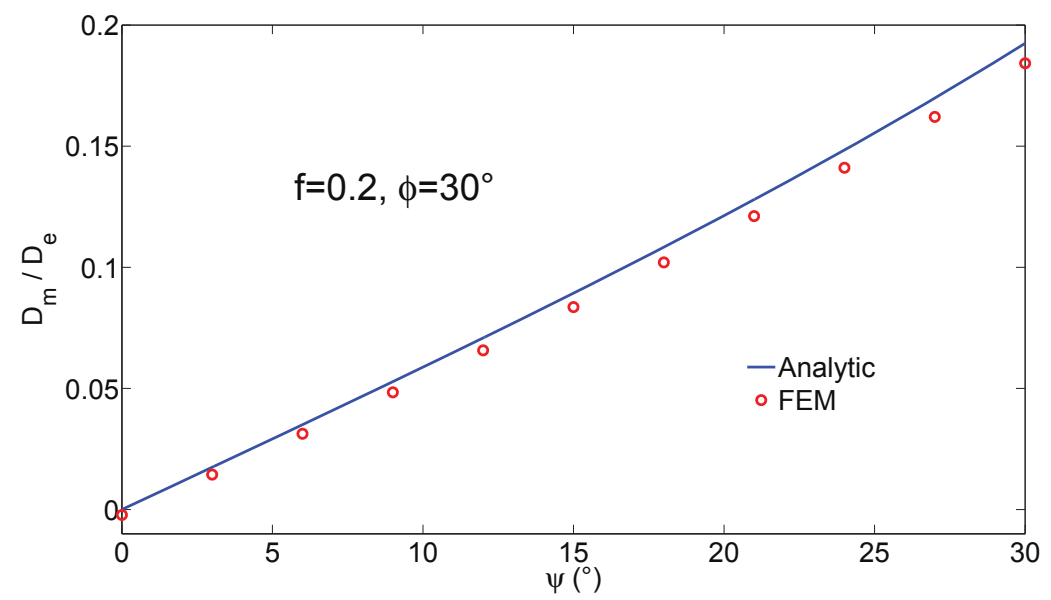

Figure 8: Comparison of plastic flow direction for pure shear loading case obtained from Eq.(68) and FEM solutions with the fixed values of porosity $f=0.2$ and friction angle $\phi=30^{\circ}$.

Finally, Fig. 9 illustrates the variation of porosity with the macroscopic stress triaxiality for a initial porosity $f=0.2$, a fixed value of friction angle $\phi=30^{\circ}$ and for three dilatancy angles $\psi=30^{\circ}, \psi=15^{\circ}$ and $\psi=5^{\circ}$. Noticeable difference between the associated case and non-associated ones is observed, particularly for high stress triaxialities.

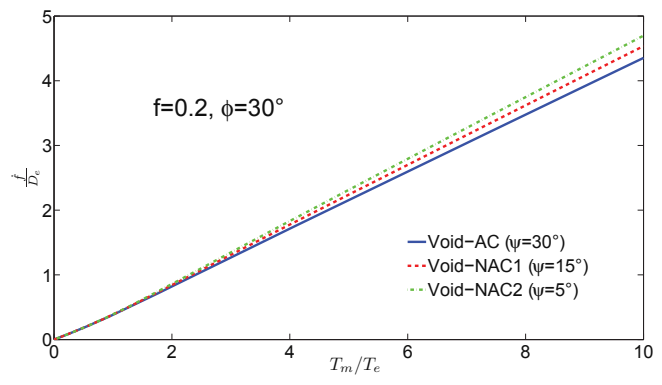

(a) Variation with positive triaxialities

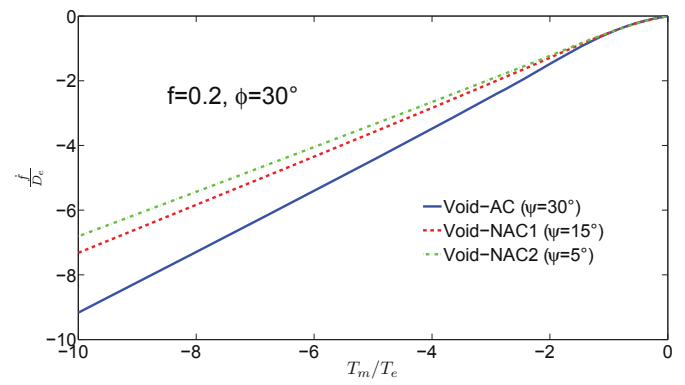

(b) Variation with negative triaxialities

Figure 9: Analytical result of the plastic void growth with a initial value of porosity $f=0.2$. Friction angle $\phi=30^{\circ}$

\section{Conclusion}

In this study, a bipotential-based variational framework of ductile porous media has been proposed. It allowed to extend classical limit analysis of porous media to the context of a matrix obeying to a non-associated flow rule. This is generally the case of various porous geomaterials or porous polymers displaying also pressure-sensitivity of the matrix. The proposed variational formulation combines the bipotential theory earlier introduced by de Saxcé et al. (1991) with homogenization techniques. It delivers closed-form expression of the macroscopic criterion for the porous materials, as well as the non-associated flow rule. 
As detailed in Section 4, application of the proposed approach to porous materials with non associated plastic matrix (characterized by a friction angle and a dilatancy angle) has led to the formulation of a macroscopic bifunctional. Minimization procedures of the bifunctional have been proposed with respect to a trial kinematically admissible velocity fields (respectively the statically admissible stress fields). This can deliver for the considered class of porous materials an upper bound (respectively lower bound) provided that the exact stress field (respectively the exact velocity field) has been adopted.

Practical implementation of the approach has been done by considering a hollow sphere subjected to uniform strain rate boundary conditions. To this end, a choice of a simple stress field together with a class of trial velocity fields has been made. The whole procedure has allowed to establish a closed form expression of the macroscopic yield function (see equations (65)), as well as the macroscopic flow rule (see equations (68)), in a parametric form. The non-associated character of the matrix (dilatancy angle) affects not only the macroscopic yield surface, but also the macroscopic flow rule which is shown to be non associated. Moreover, due to the suitable choice of the trial fields, the model also preserves the exact solution established in Cheng et al. (2012) for the hollow sphere with the non associated matrix, under pure hydrostatic loadings. It is also worth noticing that the obtained results allow to retrieve (as a particular case) the kinematically-based model proposed by (Guo et al., 2008) for the porous material with an associated Drucker-Prager matrix. This automatically includes the Gurson model for a von Mises matrix (Gurson, 1977). The predictions of the general model are fully assessed, both for the macroscopic yield surfaces and for the flow rule. To this end, numerous Finite Elements computations in non associated plasticity have been carried out on the hollow sphere. A good agreement has been observed between theoretical results and numerical data for various configurations of porosity, friction angle and dilatancy angle.

Finally, it should be noted that some improvements of the basic model are possible, in particular by searching more refined trial velocity and stress fields. The consideration of voids saturation by an internal pressure will also constitute a challenging extension which will pave the way for various applications in geomechanics including non associated poroplasticity.

\section{References}

Barthélémy, J.F., Dormieux, L., 2003. Détermination du critère de rupture macroscopique d'un milieux poreux par homogénéisation non linéaire. Comptes Rendus Mécanique, 331, 271-276.

Benzerga, A.A., Besson, J., 2001. Plastic potentials for anisotropic porous solids. European Journal of Mechanics A/Solids, 20, 397-434.

Berga, A., de Saxcé, G., 1994. Elastoplastic Finite Element Analysis of Soil Problems with Implicit Standard Material Constitutive Laws, Revue Européenne des éléments finis, 3, 411-456.

Bodovillé, G., 1999. On damage and implicit standard materials, C. R. Acad. Sci. Paris, Sér. II, Fasc. b, Méc. Phys. Astron., 327, 715-720.

Bodovillé, G., de Saxcé, G., 2001. Plasticity with non linear kinematic hardening : modelling and shakedown analysis by the bipotential approach. Eur. J. Mech., A/Solids, 20, 99-112. 
Bouby, C., de Saxcé, G., Tritsch, 2006. A comparison between analytical calculations of the shakedown load by the bipotentiel approach and step-by-step computations for elastoplastic materials with nonlinear kinematic hardening. International Journal of Solids and Structures, 43(9), 2670-2692.

Bouby, C., de Saxcé, G., Tritsch, 2009. Shakedown analysis: comparison between models with the linear unlimited, linear limited and non linear kinematic hardening. Mechanical Research Communication, 36, 556562 .

Bousshine, L., Chaaba, A., de Saxcé, G., 2001. Softening in stress-strain curve for Drucker-Prager nonassociated plasticity. Int. J. of Plasticity, 17(1), 21-46.

Bousshine, L., Chaaba, A., de Saxcé, G., 2002. Plastic limit load of plane frames with frictional contact supports. Int. J. Mech. Sci., 44, 2189-2216, 2002.

Bousshine, L., Chaaba, A., de Saxcé, G., 2003. A new approach to shakedown analysis for non-standard elastoplastic material by the bipotential. Int. J. of Plasticity, 19(5), 583-598.

Buliga, M., de Saxcé, G. Vallée, C., 2008. Existence and construction of bipotential for graphs of multivalued laws. Journal of Convex Analysis. J. Convex Analysis, 15(1), 87-104.

Buliga, M., de Saxcé, G. Vallée, C., 2009. Bipotentials for non monotone multivalued operators: fundamental results and applications. Acta Applicandae Mathematicae, 110(2), 955-972.

Buliga, M., de Saxcé, G. Vallée, C., 2009. Blurred constitutive laws and bipotential convex covers. Mathematics and Mechanics of Solid Journal. First on line.

Buliga, M., de Saxcé, G. Vallée, C., 2010. Non maximal cyclically monotone graphs and construction of a bipotential for the Coulomb's dry friction law. J. Convex Analysis, 17(1), 81-94.

Buliga, M., de Saxcé, G. Vallée, C., 2010. Blurred maximal cyclically monotone sets and bipotentials. Analysis and Applications, 8(4), 323-336.

O. Cazacu and J. B. Stewart. Plastic potentials for porous aggregates with the matrix exhibiting tensioncompression asymmetry. Journal of the Mechanics and Physics of Solids, $57: 325-341,2009$.

Chaaba, A., Bousshine, L., de Saxcé, G., 2010. Kinematic Limit Analysis of Nonassociated Perfectly Plastic Material by the Bipotential Approach and Finite Element Method. J. Appl. Mech., 77(3), 031016.

Chen, W.F., 1975. Limit Analysis and Soil Plasticity. Elsevier, New York.

Chen, W.F., Liu, X.L., 1990. Limit analysis in soil mechanics. Developments in Geotechnical Engineering, Vol. 52.

Cheng, L., Jia, Y., Oueslati, A., de Saxcé, G, Kondo, D., 2012 Plastic limit state of the hollow sphere model with non-associated Drucker-Prager material under isotropic loading. Computational Materials Science, Vol. 62, 210-215.

Cheng, L., de Saxcé, G, Kondo, D.. A stress-based variational model for ductile porous materials. Vol. 55, 133-151.

Cheng, L. Homogenization of porous media with plastic matrix and non-associated flow rule by variational methods. Ph.D. Thesis, Univ. Lille 1, 2013.

Cheng, L., Guo, T.F., 2006. Void interaction and coalescence in polymeric materials. International Journal of Solids and Structures, 44, 1787-1808.

de Buhan, P. A fundamental approach to the yield design of reinforced soil structures Chap. 2: yield design homogenization theory for periodic media, Ph.D. Thesis, Univ. Pierre et Marie Curie, Paris VI, 1986.

de Saxcé, G., Feng, Z.Q., 1991. New inequality and functional for contact friction: The implicit standard material approach. Mechanics of Structures and Machines, 19, 301-325, 1991.

de Saxcé, G., 1992. Une généralisation de l'inégalité de Fenchel et ses applications aux lois constitutives. C. R. Acad. Sci. Paris, Sér. II, 314, 125-129, 1992.

de Saxcé, G., Bousshine, L., 1993. On the extension of limit analysis theorems to the non-associated flow rules in soils and to the contact with Coulomb's friction. XI Polish Conference on Computer Methods in Mechanics. Kielce, Poland, 815-822.

de Saxcé, G., 1995. The bipotential method, a new variational and numerical treatment of the dissipative laws of materials. 10th Int. Conf. on Mathematical and Computer Modelling and Scientific Computing, Boston, Massachusetts.

de Saxcé, G., Bousshine, L., 1998. Limit Analysis Theorems for the Implicit Standard Materials: Application 
to the Unilateral Contact with Dry Friction and the Non Associated Flow Rules in Soils and Rocks. Int. J. Mech. Sci., 40(4),387-398.

de Saxcé, G., Feng, Z.Q., 1998. The bi-potential method: a constructive approach to design the complete contact law with friction and improved numerical algorithms. Mathematical and Computer Modelling, 6, 225-245, 1998.

de Saxcé, G., Bousshine, L., 2002. Implicit standard materials. D. Weichert G. Maier eds. Inelastic behaviour of structures under variable repeated loads, CISM Courses and Lectures 432, Springer, Wien.

de Saxcé, G., Fortin, J., Millet, O. 2004. About the numerical simulation of the dynamics of granular media and the definition of the mean stress tensor. Mechanics of Materials, 36, 1175-1184.

Drucker, D.C. 1953. Limit analysis of two - and three - dimensional soil mechanics problems. J. Mech. Phys.Solids. 1, 217-226.

I. Ekeland, I., Temam, R., 1975. Convex analysis and variational problems, Amsterdam, North Holland.

Fenchel, W., 1949. On conjugate convex functions. Canadian Journal of Mathematics, 1, 73-77.

Feng, Z.Q., Hjiaj, M., de Saxcé, G., Mróz, Z., 2006. Effect of frictional anisotropy on the quasistatic motion of a deformable solid sliding on a planar surface. Comput. Mech., 37, 349-361.

Feng, Z.Q., Hjiaj, M., de Saxcé, G., Mróz, Z., 2006. Influence of frictional anisotropy on contacting surfaces during loading/unloading cycles. International Journal of Non-Linear Mechanics, 41(8), 936-948.

Fine, N.J., 1988. Basic Hypergeometric Series and Applications. Amer. Math. Soc., Providence, RI.

Fortin, J., de Saxcé, G., 1999. Modlisation numrique des milieux granulaires par lapproche du bipotentiel. C.R. de lAcadmie des Sciences, Srie IIb, 327, 721-724.

Fortin, J., Hjiaj, M., de Saxcé, G., 2002. An improved discrete element method based on a variational formulation of the frictional contact law. Comput. Geotech., 29, 609-640.

Garajeu, M., Suquet, P., 1997. Effective properties of porous ideally plastic or viscoplastic materials containing rigid particles. Journal of the Mechanics and Physics of Solids, 45, 873-902.

Gologanu, M., Leblond, J.B., Perrin, G., Devaux, J., 1997. Recent extensions of Gurson's model for porous ductile metals. P. Suquet (Ed.), Continuum Micromechanics, Springer-Verlag.

Guo, T.F., Faleskog, J., Shih, C.F.2008. Continuum modeling of a porous solid with pressure-sensitive dilatant matrix. Journal of the Mechanics and Physics of Solids, 56, 2188-2212.

Gurson, A.L., 1977. Continuum theory of ductile rupture by void nucleation and growth - part I: Yield criteria and flow rules for porous ductile media, Journal of Engineering Materials and Technology, 99, $2-15$.

Halphen, B., Nguyen Quoc, S., 1975. Sur les matériaux standard généralisés. C. R. Acad. Sci. Paris, 14, 39-63.

Hjiaj, M., Bodovillé, G., de Saxcé, G., 2000. Matériaux viscoplastiques et loi de normalité implicites. C. R. Acad. Sci. Paris, Sér. II, Fasc. b, Méc. Phys. Astron., 328, 519-524.

Hjiaj, M., de Saxcé, G., Mróz, Z., 2002. A variational-inequality based formulation of the frictional contact law with a non-associated sliding rule. European Journal of Mechanics A/Solids, 21, 49-59.

Hjiaj, M., Fortin, J., de Saxcé, G., 2003. A complete stress update algorithm for the non-associated DruckerPrager model including treatment of the apex. International Journal of Engineering Science, 41, 1109-1143.

Hjiaj, M., Feng, Z.Q., de Saxcé, G., Mróz, Z., 2004. Three dimensional finite element computations for frictional contact problems with on-associated sliding rule. Int. J. Numer. Methods Eng., 60, 2045-2076.

Jeong, H.Y., Pan, J., 1995. A macroscopic constitutive law for porous solids with pressure-sensitive matrices and its applications to plastic flow localization. Journal of the Mechanics and Physics of Solids, 39, 1385-1403.

Jeong, H.Y., 2002. A new yield function and a hydrostatic stress-controlled model for porous solids with pressure-sensitive matrices. Journal of the Mechanics and Physics of Solids, 32, 3669-3691.

Keralavarma, S.M., Benzerga, A.A. 2010. A constitutive model for plastically anisotropic solids with nonspherical voids. Journal of the Mechanics and Physics of Solids, 58, 874-901.

Laborde, P., Renard, Y., 2008. Fixed points strategies for elastostatic frictional contact problems. Math. Meth. Appl. Sci., 31, 415-441.

Ladevèze, P., 1975. Comparaisons de modèles de milieux continus, Thèse d'Etat, Université Pierre et Marie 
Curie, Paris.

Ladevèze, P., Coffignal, G., Pelle, J.P., 1986. Accuracy of elastoplastic and dynamic analysis. in: I. Babuska, J. Gago, E. Oliveira and O.C. Zienkiewicz, eds., Accuracy Estimates and Adaptative Refinements in Finite Element Computations, John Wiley, 181-203.

Ladevèze, P., Pelle, J.P., Rougeot, P., 1991. Error estimation and mesh optimization for classical finite element. Engrg. Comput. 8, 69-80.

Ladevèze, P., Moes, N., 1997. A new a posteriori error estimation for nonlinear time-dependent finite element analysis. Comput. Methods. Appl. Mech. Engrg., 157, 45-68.

Ladevèze, P., Pelle, J.P., 2001. La maîtrise du calcul en mécanique linéaire et non linéaire. Hermes Science.

Ladevèze, P., Florentin, E., 2006. Verification of stochastic models in uncertain environments using the constitutive relation error method. Comput. Methods. Appl. Mech. Engrg., 196, 225-224.

Ladevèze, P., Puel, G., Deraemaeker, A., Romeuf, T., 2006. Validation of structural dynamics models containing uncertainties. Comput. Methods. Appl. Mech. Engrg., 195, 373-393.

J. Lin, J.-F. Shao, D. Kondo, 2011. A two scale model of porous rocks with DruckerPrager matrix: Application to a sandstone. Mechanics Research Communications 38 (2011) 602606

J. Lin, S.Y. Xie, J.F. Shao and D. Kondo. A micromechanical modeling of ductile behavior of a porous chalk: Formulation, identification, and validation. International Journal for Numerical and Analytical Methods in Geomechanics. 36, (2012) 1245-1263.

Madou, K., Leblond, J.B., 2012. A Gurson-type criterion for porous ductile solids containing arbitrary ellipsoidal voids I: Limit-analysis of some representative cell. Journal of the Mechanics and Physics of Solids, 60, 1020-1036.

Madou, K., Leblond, J.B., 2012. A Gurson-type criterion for porous ductile solids containing arbitrary ellipsoidal voids II: Determination of yield criterion parameters. Journal of the Mechanics and Physics of Solids, 60, 1037-1058.

Maghous, S., Dormieux, L., Barthélémy, J.F., 2009. Micromechanical approach to the strength propoerties of frictional geomaterials. European Journal of Mechanics A/Solids, 28, 179-188.

Magnier, V., Charkaluk, E., Bouby, C., de Saxcé, G., 2006. Bipotential Versus Return Mapping Algorithms: Implementation of Non-Associated Flow Rules, in Proceedings of The Eighth International Conference on Computational Structures Technology (las Palmas de Gran Canaria, sept. 12-15, 2006), B.H.V. Topping, G. Montero and R. Montenegro, (Editors), Civil-Comp Press, Stirlingshire, United Kingdom, paper 68.

Monchiet, V., Charkaluk, E., Kondo, D., 2007. An improvment of Gurson-type models of porous materials by Eshelby-like trial velocity fields. Comptes Rendus Mécanique, 335, 32-41.

Monchiet, V., Cazacu, O., Kondo, D., 2008. Macroscopic yield criteria for plastic anisotropic materials containing spheroidal voids. International Journal of Plasticity, 24, 1158-1189.

Monchiet, V., Kondo, D., 2013. Combined voids size and shape effects on the macroscopic criterion of ductile nanoporous materials. International Journal of Plasticity, 43, 20-41.

Moreau, J.J.,2003. Fonctionnelles convexes. Istituto Poligrafico e Zecca dello Stato, Rome.

Parmer, A.C., 1973. Proceedings of the Symposium on the Role of Plasticity in Soil Mechanics. Cambridge University, Cambridge, England, 314 pp.

Ponte Castaneda, P., 1991. The effective mechanical properties of nonlinear isotropic composites. J. Mech. Phys. Solids, 39, 45-71.

Radenkovic, D., 1961. Limit analysis theorems for a Coulomb material with a non standard dilatation. C. R. Acad. Sci. Paris, 252, 4103-4104.

Rockafellar, R.T., 1970. Convex Analysis, Princeton University Press, Princeton.

Salenon, Calcul la rupture et analyse limite, Presses de lENPC, 1983.

Save, M.A., Massonnet, C.E., de Saxcé, G., 1997. Plastic limit analysis of plates, shells and disks. Elsevier, New York.

Shen, W.Q., Shao,J-F, Kondo, D., Gatmiri, B., 2012. A micromacro model for clayey rocks with a plastic compressible porous matrix. International Journal of Plasticity, 36, 64-85.

Suquet, P. Plasticité et homogénéisation. Ph.D. Thesis, Univ. Pierre et Marie Curie, Paris VI, 1982.

Suquet, P., 1995. Overall properties of nonlinear composites: a modified secant moduli approach and its 
link with Ponte Castaneda's nonlinear variational procedure. C. R. Acad. Sc. Paris, IIb, 320, 563-571.

Telega, J.J., 2002. Extremum principles for nonpotential and initial-value problems. Arch. Mech., 54, 565592.

Telega, J.J., Mohammed, H., Sloan, S.W., 2004. An of Limit Analysis Theorems to Incompressible Material with a Non-Associated Flow Rule. Complementarity, Duality and Symmetry in Nonlinear Mechanics, Advances in Mechanics and Mathematics Volume 6, pp 255-275

Thoré, P., Pastor, F., Pastor, J., Kondo, D., 2009. Closed-form solutions for the hollow sphere model with Coulomb and Drucker-Prager materials under isotropic loadings. Comptes Rendus Mécanique, 337, 260-267.

Vallée, C., Lerintiu, C., Fortuné, D., Ban, M., de Saxcé, G., 2005. Hill's bipotential. M. Mihailescu-Suliciu eds. New Trends in Continuum Mechanics, Theta Series in Advanced Mathematics, Theta Foundation, Bucarest, Roumania, 339-351.

Boulbibane, M., Weichert, D., 1997. Application of shakedown theory to soils with non-associated flow rules. Mechanics Research Communications. 24, 513-519.

Zouain, N., Pontes Filho, I., Borges, L., Mouta da Costa, L., 2007. Plastic collapse in non-associated hardening materials with application to Cam-clay. International Journal of Solids and Structures, 44, 4382-4398.

Zouain, N., Pontes Filho, I., Vaunat, J., 2010. Potentials for the modified Cam-Clay model. European Journal of Mechanics - A/Solids, 29, 327-336.

\section{Appendix A. Explicit expressions of $\Pi, \hat{\Pi}$ and the derivatives $\Pi_{, C_{0}}, \Pi_{, D_{e}}, \hat{\Pi}_{C_{0}}$, $\hat{\Pi}_{, D_{e}}$}

In order to explicitly express the closed-form macroscopic criterion (65), we provide as follows the expressions of $\Pi$ and $\hat{\Pi}$ and their partial derivatives with respect to $C_{0}$ and $D_{e}$.

Eqs. (54) for $\Pi(\boldsymbol{v})$ and $(58)$ for $I(\gamma)$ simultaneously contain the term $K(\xi)$, which is smooth over the compactly supported domain with extreme values $K_{\max }=K(0)=1$ and $K_{\text {min }}=K(-1)=0.962$. Following (Gurson, 1977), this function is taken to be unity for simplicity of calculation; that reduces (54) and (58) to:

$$
\begin{gathered}
\Pi(\boldsymbol{v})=D_{e} \int_{f}^{1} \sqrt{1+\tau^{2} x^{-2 / \tilde{s}}} d x, \\
I(\gamma)=D_{e} \int_{f}^{1} x^{-\gamma} \sqrt{1+\tau^{2} x^{-2 / \tilde{s}}} d x .
\end{gathered}
$$

Additionally, Eqs.(A.1), (A.2) and the derivatives $\Pi_{, C_{0}}, \Pi_{, D_{e}}, \hat{\Pi}_{C_{0}}$ and $\hat{\Pi}_{, D_{e}}$ can not be calculated into simple forms. Fortunately, they can be expressed by means of the Gauss hypergeometric function (see for example Fine (1988)) defined by:

$$
{ }_{2} F_{1}(a, b ; c ; z)=\frac{\Gamma(c)}{\Gamma(b) \Gamma(c-b)} \int_{0}^{1} \frac{t^{b-1}(1-t)^{c-b-1}}{(1-t z)^{a}} d t
$$

This function is a solution of the hypergeometric differential equation

$$
z(1-z) y^{\prime \prime}+[c-(a+b+1) z] y^{\prime}-a b y=0
$$


The regular solution is classically written in the form of the following power series

$$
{ }_{2} F_{1}(a, b ; c ; z)=\sum_{n=0}^{\infty} \frac{(a)_{n}(b)_{n}}{(c)_{n}} \frac{z^{n}}{n !},
$$

where $(a)_{n}$ is the Pochhammer symbol defined by

$$
(a)_{n}=\frac{\Gamma(a+n)}{\Gamma(a)}=a(a+1) \ldots(a+n-1)
$$

Let us introduce that

$$
\iota=\frac{\tau}{f^{1 / \tilde{s}}}
$$

$\Pi$ and $I$ (Eqs. (A.1) and (A.2)) can be indirectly expressed as follows:

$$
\begin{gathered}
\mathcal{P}=\frac{\Pi}{D_{e}}={ }_{2} F_{1}\left(-\frac{1}{2},-\frac{\tilde{s}}{2} ; 1-\frac{\tilde{s}}{2} ;-\tau^{2}\right)-f \cdot_{2} F_{1}\left(-\frac{1}{2},-\frac{\tilde{s}}{2} ; 1-\frac{\tilde{s}}{2} ;-\iota^{2}\right) \\
\mathcal{Q}=\frac{I}{D_{e}}=s^{-1}\left[{ }_{2} F_{1}\left(-\frac{1}{2},-\frac{\tilde{s} s^{-1}}{2} ; 1--\frac{\tilde{s} s^{-1}}{2} ;-\tau^{2}\right)\right. \\
\left.-f^{s^{-1}} \cdot{ }_{2} F_{1}\left(-\frac{1}{2},-\frac{\tilde{s} s^{-1}}{2} ; 1--\frac{\tilde{s} s^{-1}}{2} ;-\iota^{2}\right)\right]
\end{gathered}
$$

Finally, considering (A.1), (A.2) and (57), the derivatives $\Pi_{C_{0}}, \Pi_{, D_{e}}, \hat{\Pi}_{, C_{0}}$ and $\hat{\Pi}_{, D_{e}}$ can be also computed as follows:

$$
\begin{gathered}
\Pi_{C_{0}}=\frac{\tau}{\frac{\tilde{s}}{2}-1}\left[{ }_{2} F_{1}\left(\frac{1}{2}, 1-\frac{\tilde{s}}{2} ; 2-\frac{\tilde{s}}{2} ;-\tau^{2}\right)-f^{1-2 / \tilde{s}} \cdot{ }_{2} F_{1}\left(\frac{1}{2}, 1-\frac{\tilde{s}}{2} ; 2-\frac{\tilde{s}}{2} ;-\iota^{2}\right)\right] \\
\Pi_{D_{e}}={ }_{2} F_{1}\left(\frac{1}{2},-\frac{\tilde{s}}{2} ; 1-\frac{\tilde{s}}{2} ;-\tau^{2}\right)-f \cdot{ }_{2} F_{1}\left(\frac{1}{2},-\frac{\tilde{s}}{2} ; 1-\frac{\tilde{s}}{2} ;-\iota^{2}\right), \\
\hat{\Pi}_{C_{0}}=\Pi_{C_{0}}+\frac{3 \alpha T_{m}}{1-f^{\gamma}} \cdot\left(\frac{f^{\gamma}}{s} \cdot I_{,_{C_{0}}}-\Pi_{C_{0}}\right) \\
\hat{\Pi}_{D_{e}}=\Pi_{D_{e}}+\frac{3 \alpha T_{m}}{1-f^{\gamma}} \cdot\left(\frac{f^{\gamma}}{s} \cdot I_{D_{e}}-\Pi_{D_{e}}\right)
\end{gathered}
$$

where $I_{, C_{0}}$ and $I_{, D_{e}}$ have the following expressions

$$
\begin{array}{r}
I_{{ }_{C_{0}}}=\frac{2 \tau}{\tilde{s}\left(\frac{1}{s}-\frac{2}{\tilde{s}}\right)} \cdot\left[{ }_{2} F_{1}\left(\frac{1}{2},-\frac{\tilde{s}}{2}\left(\frac{1}{s}-\frac{2}{\tilde{s}}\right) ; 1-\frac{\tilde{s}}{2}\left(\frac{1}{s}-\frac{2}{\tilde{s}}\right) ;-\tau^{2}\right)\right. \\
\left.-f^{\frac{1}{s}-\frac{2}{\tilde{s}}} \cdot{ }_{2} F_{1}\left(\frac{1}{2},-\frac{\tilde{s}}{2}\left(\frac{1}{s}-\frac{2}{\tilde{s}}\right) ; 1-\frac{\tilde{s}}{2}\left(\frac{1}{s}-\frac{2}{\tilde{s}}\right) ;-\iota^{2}\right)\right] \\
I_{, D_{e}}=s \cdot\left[{ }_{2} F_{1}\left(\frac{1}{2},-\frac{\tilde{s}}{2 s} ; 1-\frac{\tilde{s}}{2 s} ;-\tau^{2}\right)-f^{\frac{1}{s}} \cdot{ }_{2} F_{1}\left(\frac{1}{2},-\frac{\tilde{s}}{2 s} ; 1-\frac{\tilde{s}}{2 s} ;-\iota^{2}\right)\right]
\end{array}
$$




\section{Appendix B. Macroscopic criterion of ductile porous media with an associated Drucker-Prager matrix}

We apply here the general procedure proposed in sections 4 and 5 to the particular case of the associated Drucker-Prager matrix $(\psi=\phi \neq 0$ and $\beta=\alpha \neq 0)$. This is the case already studied by Guo et al. (2008) by using the classical kinematical limit analysis approach which is retrieve here from the proposed variational formulation.

In this case, the associated flow rule reads:

$$
\mathbf{d}=d_{e q} \frac{\partial F}{\partial \sigma}=d_{e q}\left(\frac{3 s}{2 \sigma_{e}}+\alpha \mathbf{1}\right)
$$

The volumetric plastic strain is such that:

$$
d_{m}=\frac{1}{3} \operatorname{tr} \mathbf{d}=\alpha d_{e q}
$$

The plastic flow rule (B.1) is completed at the apex by the condition:

$$
H(\mathbf{d})=\alpha d_{e q}-d_{m} \leq 0
$$

The finite valued bipotential is reduced into:

$$
b(\boldsymbol{d}, \boldsymbol{\sigma})=\left\{\begin{array}{ll}
\frac{\sigma_{0}}{\alpha} d_{m} & \text { if } F(\boldsymbol{\sigma}) \leq 0 \quad \text { and } \quad H(\boldsymbol{d}) \leq 0 \\
+\infty & \text { otherwise }
\end{array}\right\}
$$

It should be emphasized that the bipotential in this case takes the same expression as the support function for the associated Drucker-Prager model (see for instance by Salençon (1983)). Moreover, considering the velocity field (42) in the case of associated matrix (see Guo et al. (2008))

$$
\boldsymbol{v}=C_{0}\left(\frac{b}{r}\right)^{3 / s}\left(\rho \boldsymbol{e}_{\rho}+z \boldsymbol{e}_{z}\right)+C_{1} \rho \boldsymbol{e}_{\rho}+C_{2} z \boldsymbol{e}_{z},
$$

the microscopic mean strain rate (46) and the equivalent one (47) can be recast into:

$$
d_{m}(r)=\left(1-\frac{1}{s}\right) C_{0}\left(\frac{b}{r}\right)^{3 / s}+\frac{1}{3}\left(2 C_{1}+C_{2}\right)
$$

and

$$
d_{e q}(r)=\frac{2}{3} \sqrt{\left(C_{1}-C_{2}\right)^{2}+\left(C_{1}-C_{2}\right) \frac{3 C_{0}}{s}\left(\frac{b}{r}\right)^{3 / s}\left(3 \cos ^{2} \theta-1\right)+\left(\frac{3 C_{0}}{s}\right)^{2}\left(\frac{b}{r}\right)^{6 / s}}
$$

which obviously do not comply with (B.3) everywhere in the hollow sphere.

Due to this difficulty, the idea is to relax the admissibility condition in an average sense by imposing

$$
\int_{\Omega_{M}} H(d) d V=\int_{\Omega_{M}}\left(\alpha d_{e q}-d_{m}\right) d V=0
$$


Next, by introducing (B.6) and (B.4) into (24), the macroscopic bifunctional in the case of an associated matrix can be written as:

$$
B_{0}(\boldsymbol{v}, \boldsymbol{\sigma})=\frac{1}{|\Omega|} \int_{\Omega_{M}} \frac{\sigma_{0}}{\alpha} d_{m} d V-\boldsymbol{D}: \boldsymbol{\Sigma} .
$$

with the relaxed admissibility constraint (B.8): $\int_{\Omega_{M}} d_{m} d V=\int_{\Omega_{M}} \alpha d_{e q} d V$.

For the first minimization problem of (25), as for the non associated case, it is suggested to introduce the normalized Lagrangian $\overline{\mathcal{L}}$ by omitting $\sigma_{0}$ (or taking it equal to 1 ), with $\bar{\Lambda}$ the Lagrange multiplier which is assumed constant:

$$
\overline{\mathcal{L}}=\frac{1}{|\Omega|} \int_{\Omega_{m}} \frac{d_{m}}{\alpha} d V-\mathbf{T}: \mathbf{D}+\bar{\Lambda}\left[\int_{\Omega_{M}}\left(\alpha d_{e q}-d_{m}\right) d V\right]
$$

Putting (B.6) and (B.7) into (B.10) leads to:

$$
\bar{L}=\alpha \bar{\Lambda} \Pi+\left(\frac{1}{\alpha}-\bar{\Lambda}\right)\left[(1-f) D_{m}-\left(f^{\gamma}-f\right) C_{0}\right]-\left(3 T_{m} D_{m}+T_{e} D_{e}\right)
$$

The following minimization relations are obtained:

$$
\left\{\begin{array}{l}
\frac{\partial \bar{L}}{\partial C_{0}}=\alpha \bar{\Lambda} \frac{\partial \Pi}{\partial C_{0}}-\left(\frac{1}{\alpha}-\bar{\Lambda}\right)\left(f^{\gamma}-f\right)=0 \\
\frac{\partial \bar{L}}{\partial D_{m}}=\left(\frac{1}{\alpha}-\bar{\Lambda}\right)(1-f)-3 T_{m}=0 \\
\frac{\partial \bar{L}}{\partial D_{e}}=\alpha \bar{\Lambda} \frac{\partial \Pi}{\partial D_{e}}-T_{e}=0
\end{array}\right.
$$

The first equation of (B.12) delivers the optimal expression of $\bar{\Lambda}$

$$
\bar{\Lambda}=\frac{\frac{1}{\alpha}\left(f^{\gamma}-f\right)}{f^{\gamma}-f+\alpha \frac{\partial \Pi}{\partial C_{0}}}
$$

which, when reported in the two last ones, leads to the expression of the macroscopic admissible stress components, the parametric expression of the macroscopic criterion then reads:

$$
\begin{gathered}
3 T_{m}=(1-f)\left[\frac{1}{\alpha}-\frac{\frac{1}{\alpha}\left(f^{\gamma}-f\right)}{f^{\gamma}-f+\alpha \frac{\partial \Pi}{\partial C_{0}}}\right]=\frac{(1-f) \frac{\partial \Pi}{\partial C_{0}}}{f^{\gamma}-f+\alpha \frac{\partial \Pi}{\partial C_{0}}} \\
T_{e}=\frac{\left(f^{\gamma}-f\right) \frac{\partial \Pi}{\partial D_{e}}}{f^{\gamma}-f+\alpha \frac{\partial \Pi}{\partial C_{0}}}
\end{gathered}
$$

which corresponds to the result obtained by Guo et al. (2008). It is worth noticing that, due to the relaxation of the admissibility condition, (B.14) and (B.15) do not guarantee the upper bound character of the result. 


\section{Appendix C. Complementary results concerning the effects of the porosity, fric- tion angle and dilatancy angle on the macroscopic criterion}

In this section, we will illustrate the influences of the porosity $f$ and the friction angle $\phi$, together with that of the dilatancy angle $\psi$. The analytical results described in Appendix C.1 will be validated from the FEM computations shown in Appendix C.2.

\section{Appendix C.1. Analytical results}

Fig.C.10 displays the yield surfaces with a relatively smaller porosity $f=0.15$ and a bigger one $f=0.25$, both for the associated case (AC) and two non-associated cases (NAC1 and NAC2). Moreover, the same values of material parameters as described in subsection 7.1 (for the case $f=0.2$ ) are respectively adopted for the three cases. As observed through this figure, the yield loci of non-associated cases are lower than the corresponding associated one. It is interesting to point out that the difference between the yield surfaces of the associated case and non-associated one becomes smaller when the porosity is bigger.

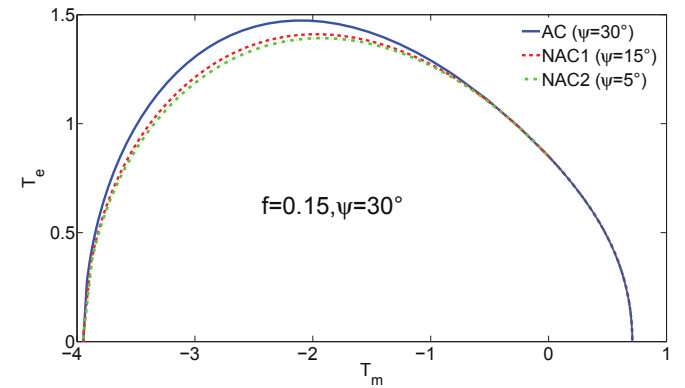

(a) Porosity: $f=0.15$, friction angle $\phi=30^{\circ}$

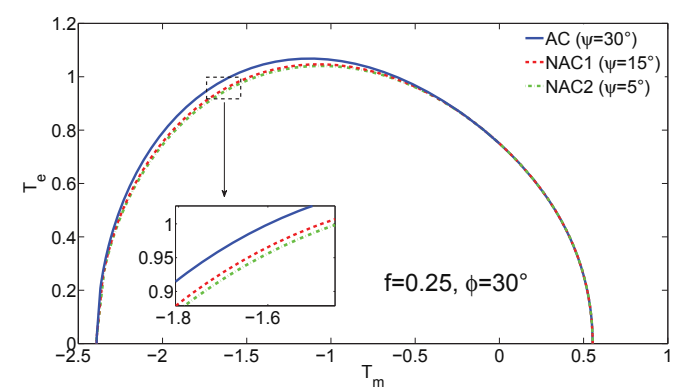

(b) Porosity: $f=0.25$, friction angle $\phi=30^{\circ}$

Figure C.10: Comparison of yield surfaces illustrated from (65) between the associated case (denoted AC) with dilatancy angle $\psi=30^{\circ}$ and two non-associated cases (denoted NAC1 and NAC2) with $\psi=15^{\circ}$ and $5^{\circ}$, respectively.

Next, in order to estimate the influence of the friction angle $\phi$ on the proposed macroscopic criterion (65), two groups of comparisons with a fixed value of porosity $f=0.2$ but different friction angles $\phi=20^{\circ}$ and $40^{\circ}$, are reported on Fig.C.11. More specifically, for $\phi=20^{\circ}$, the non-associated case NAC1 and NAC2 are defined by $\psi=15^{\circ}$ and $\psi=10^{\circ}$. For the case of $\phi=40^{\circ}$, the values $\psi=30^{\circ}$ and $\psi=20^{\circ}$ are considered. As shown on figure (65), it is observed that the yield locus of non-associated case decreases with the decrease of $\psi$. Additionally, from this group of comparisons, it is observed that the difference between the associated yield surface and the non-associated one diminishes when the value of friction angle $\phi$ is smaller ${ }^{4}$.

\footnotetext{
${ }^{4}$ For the same reductions of dilatancy angle $\psi$ with respect to the associated one.
} 

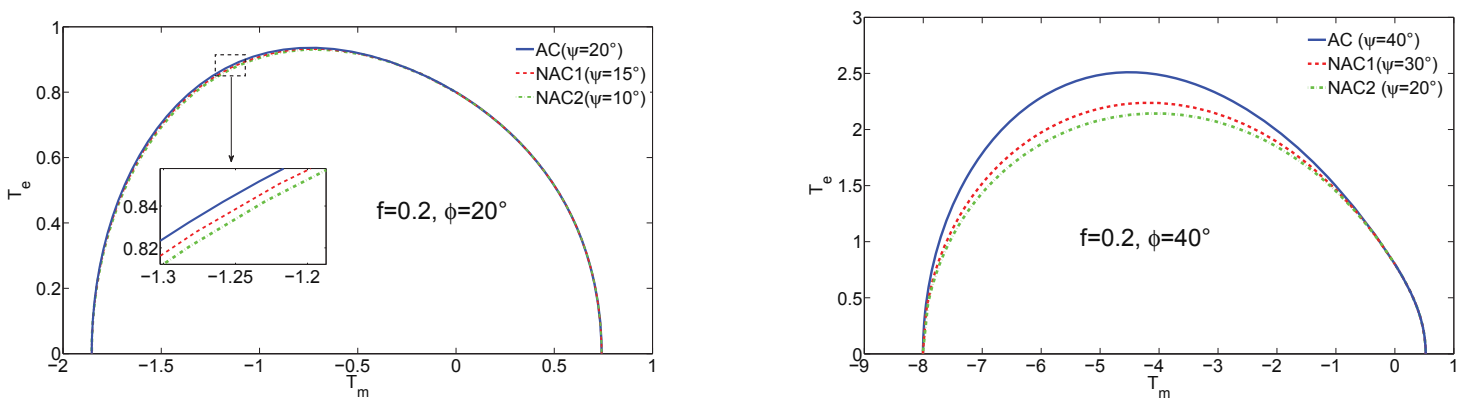

(a) AC: $\phi=\psi=20^{\circ}$; NAC1: $\phi=20^{\circ}, \psi=15^{\circ}$; (b) AC: $\phi=\psi=40^{\circ}$; NAC1: $\phi=40^{\circ}, \psi=30^{\circ}$; NAC2: $\phi=20^{\circ}, \psi=10^{\circ}$

NAC2: $\phi=20^{\circ}, \psi=20^{\circ}$

Figure C.11: Comparison of plastic flow direction for pure shear loading case obtained from Eq.(69) and FEM solutions.

\section{Appendix C.2. Assessment of analytical results with FEM computations}

The influence of the porosity $f$ on the plastic limit states (directions of plastic flow and limit stresses) are reported on Figs.C.12 and C.13. It is observed that the difference between the non-associated case and the associated one is more significant for $f=0.15$ in the compression zone $\left(T_{m}=\Sigma_{m} / \sigma_{0}<0\right)$ than for the case $f=0.25$. This fact numerically confirms that, as in Appendix C.1, the difference of the yield locus between the associated case and the non associated one is smaller with the decrease of the friction angle $\phi$.

Finally, the influence of friction angle $\phi$ is assessed through Figs.C.14 and C.15 by adopting a fixed value of porosity $f=0.2$ and two values of friction angle $\phi=20^{\circ}$ and $40^{\circ}$, respectively. From the illustrations on Figs. $14(\mathrm{~b})$ and $15(\mathrm{~b})^{5}$, it is validated that the difference between non-associated case with a fixed dilatancy angle $\psi$ and corresponding associated one is negligible when the friction angle $\phi$ is adequately smaller.

It is important to point out that for the case $\phi=40^{\circ}$, the FEM yield surfaces are relatively higher than the analytical one; this probable results from the proposed stress fields, for which the internal boundary condition is relaxed because of its homogeneous part.

\footnotetext{
${ }^{5}$ For the non-associated cases with material parameters $\phi=40^{\circ}, \psi=30^{\circ}$ and $20^{\circ}$, there are not enough FEM results to represent the plastic flow and to construct the yield surface due to the so strong nonlinearity of the non-associated model that the corresponding FEM plastic limit states cannot be obtained in this paper.
} 


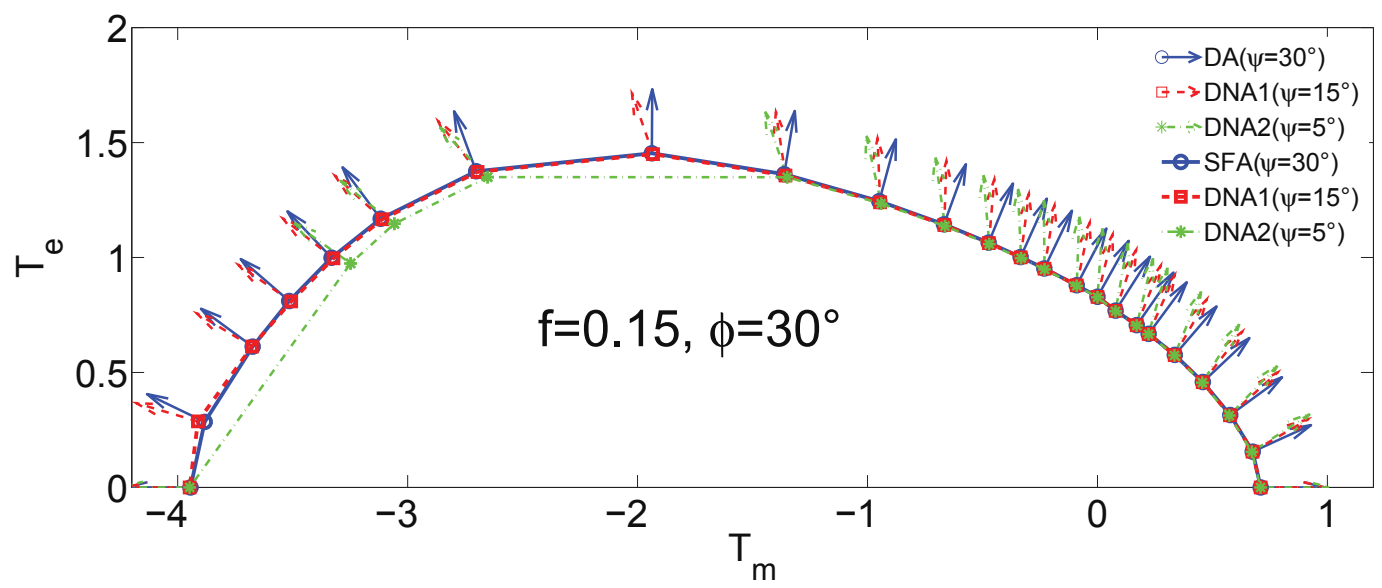

(a) Illustration of FEM results: plastic flow directions (denoted DA for associated case, DNA1 and DNA2 for non-associated ones) and yield surface (denoted SFA for associated case, SFNA1 and SFNA2 for nonassociated ones).

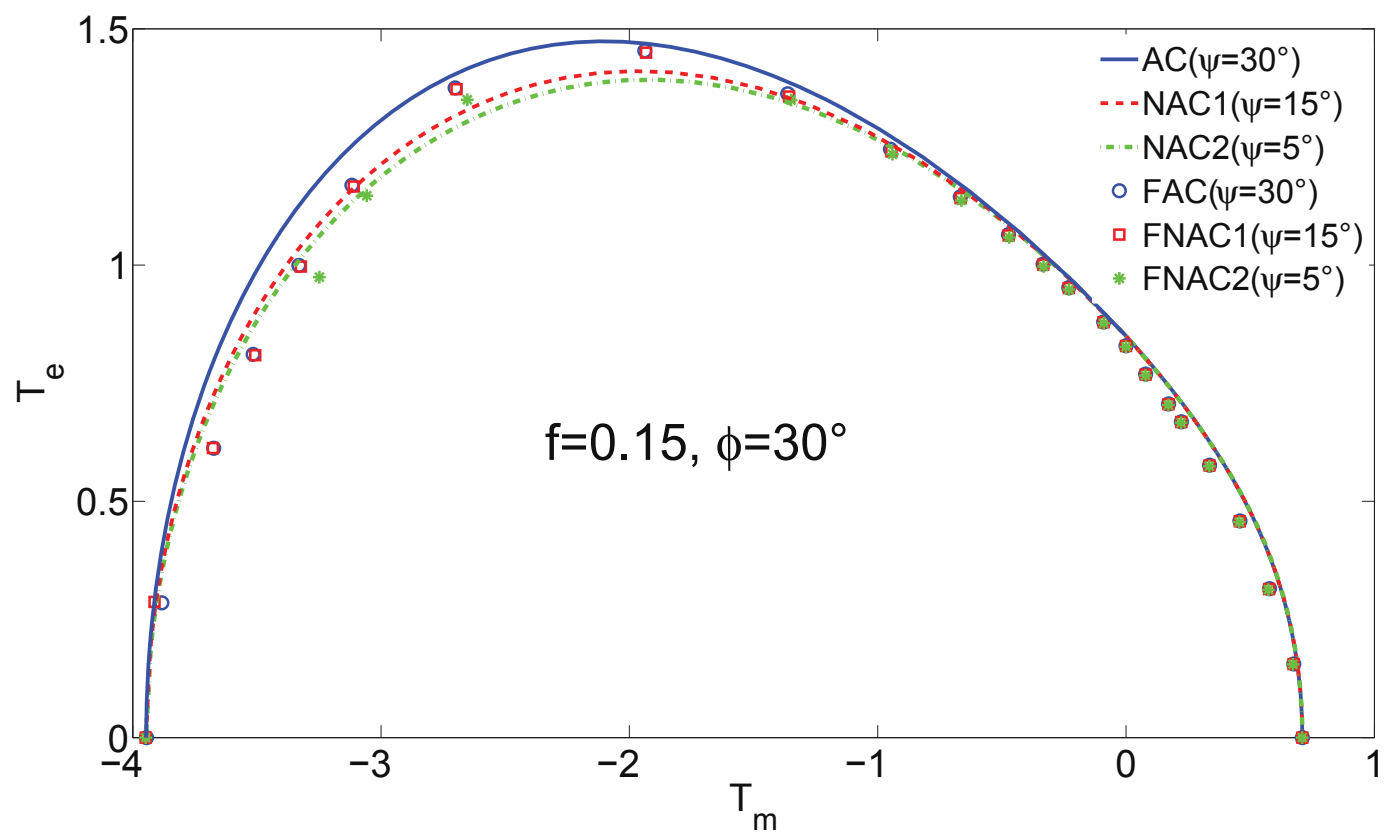

(b) Comparison between the yield surfaces obtained from the established criteria (65) and FEM results (denoted FAC for associated case, FAC1 and FAC2 for non-associated ones).

Figure C.12: Numerical validation for the established model with fixed porosity $f=0.15$ and friction angle $\phi=30^{\circ}$. 


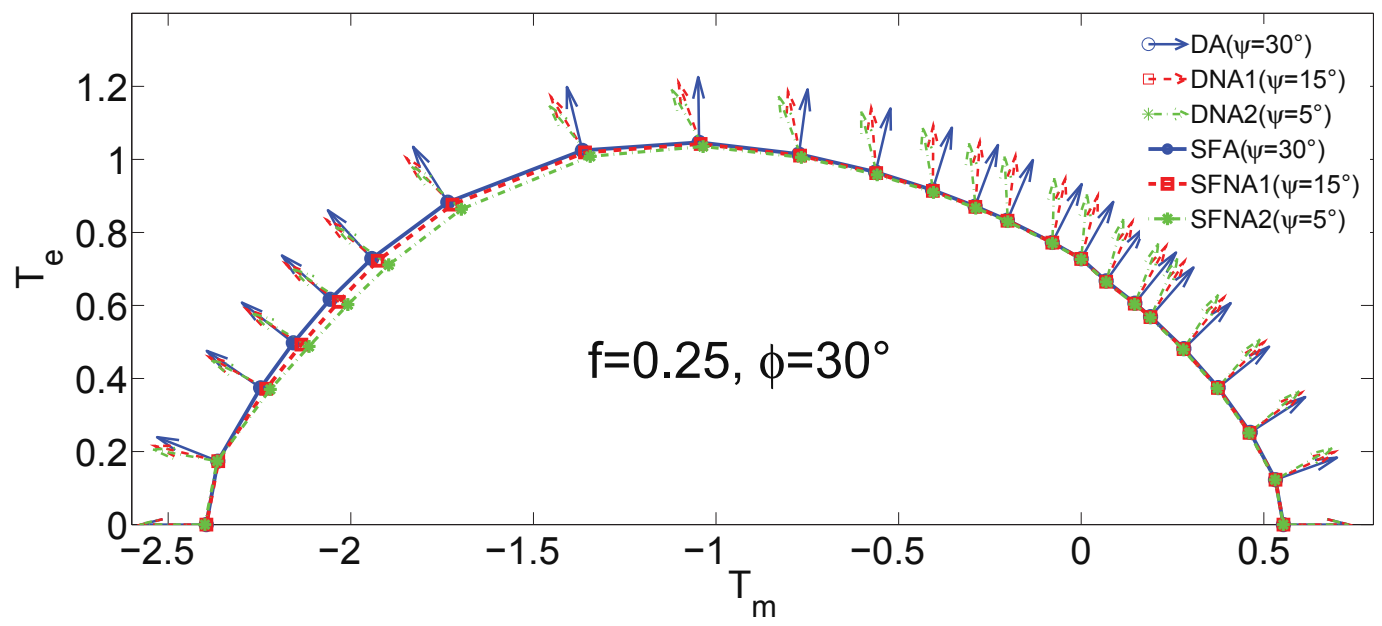

(a) Illustration of FEM results: plastic flow directions (denoted DA for associated case, DNA1 and DNA2 for non-associated ones) and yield surface (denoted SFA for associated case, SFNA1 and SFNA2 for nonassociated ones).

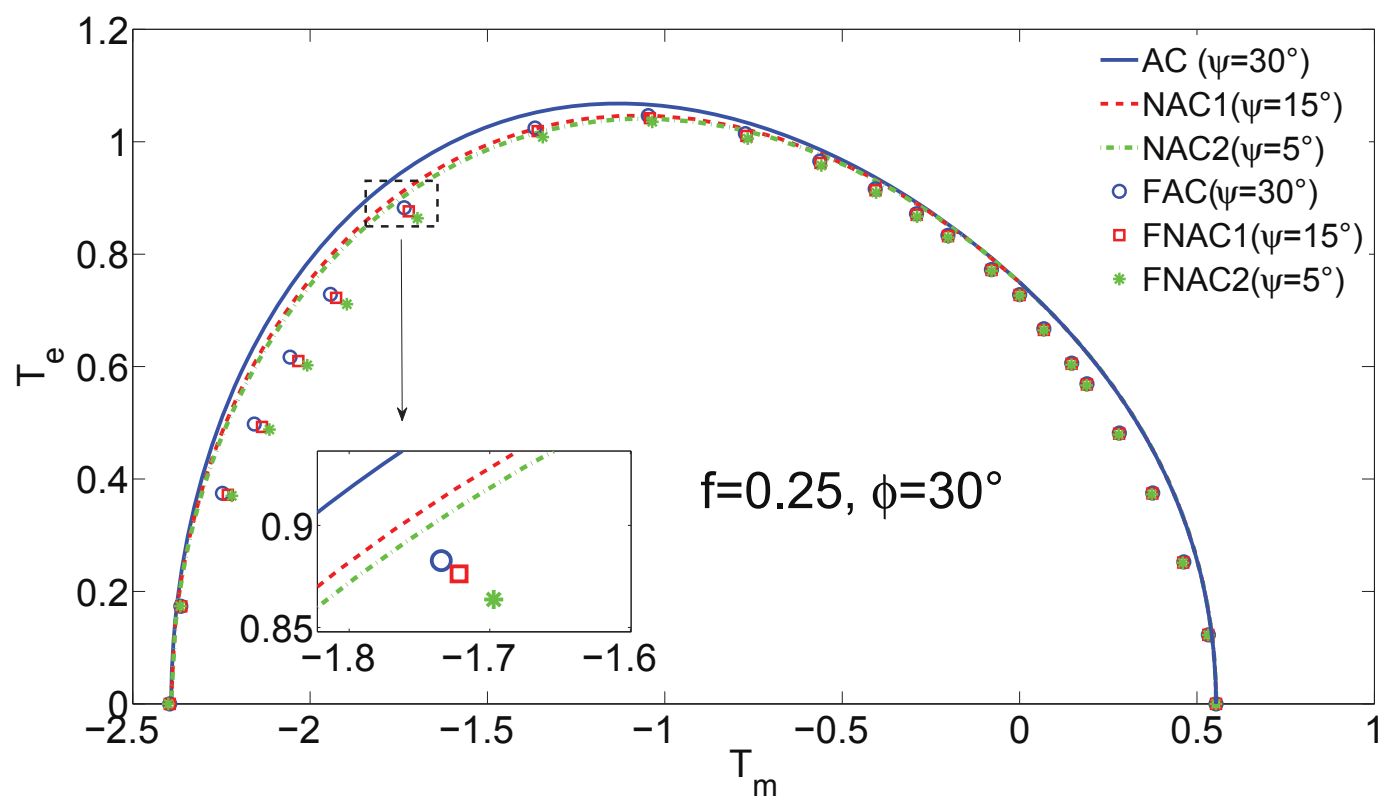

(b) Comparison between the yield surfaces obtained from the established criteria (65) and FEM results (denoted FAC for associated case, FAC1 and FAC2 for non-associated ones).

Figure C.13: Numerical validation for the established model with fixed porosity $f=0.25$ and friction angle $\phi=30^{\circ}$. 


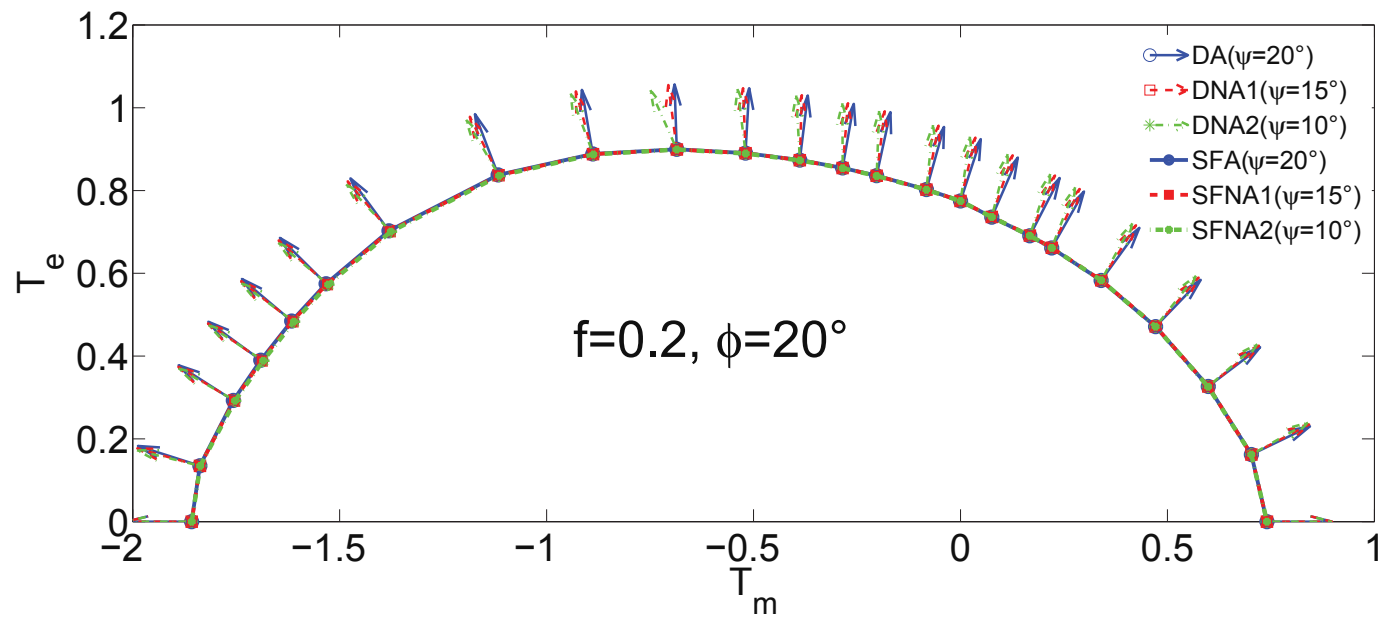

(a) Illustration of FEM results: plastic flow directions (denoted DA for associated case, DNA1 and DNA2 for non-associated ones) and yield surface (denoted SFA for associated case, SFNA1 and SFNA2 for nonassociated ones).

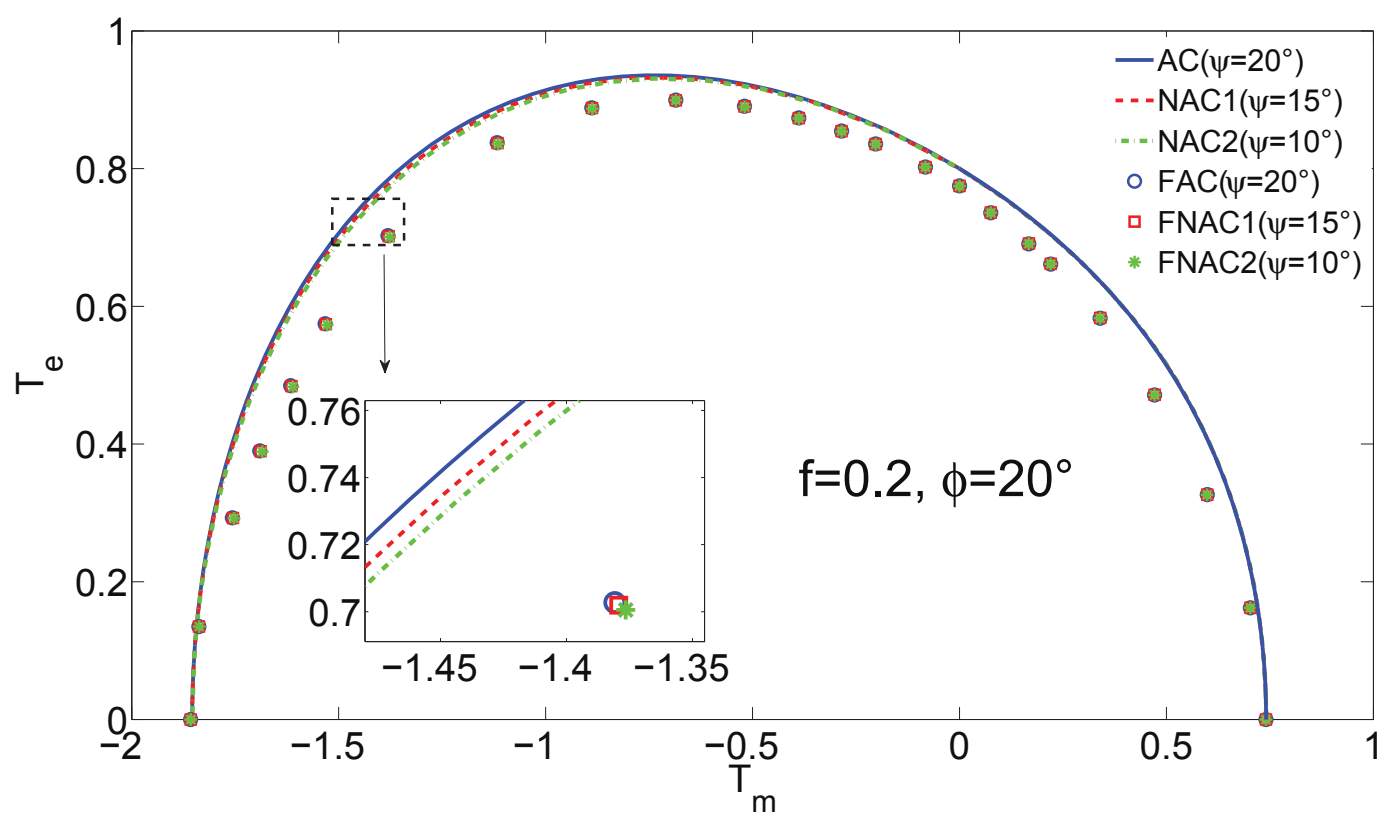

(b) Comparison between the yield surfaces obtained from the established criteria (65) and FEM results (denoted FAC for associated case, FAC1 and FAC2 for non-associated ones).

Figure C.14: Numerical validation for the established model with fixed porosity $f=0.2$ and friction angle $\phi=20^{\circ}$. 


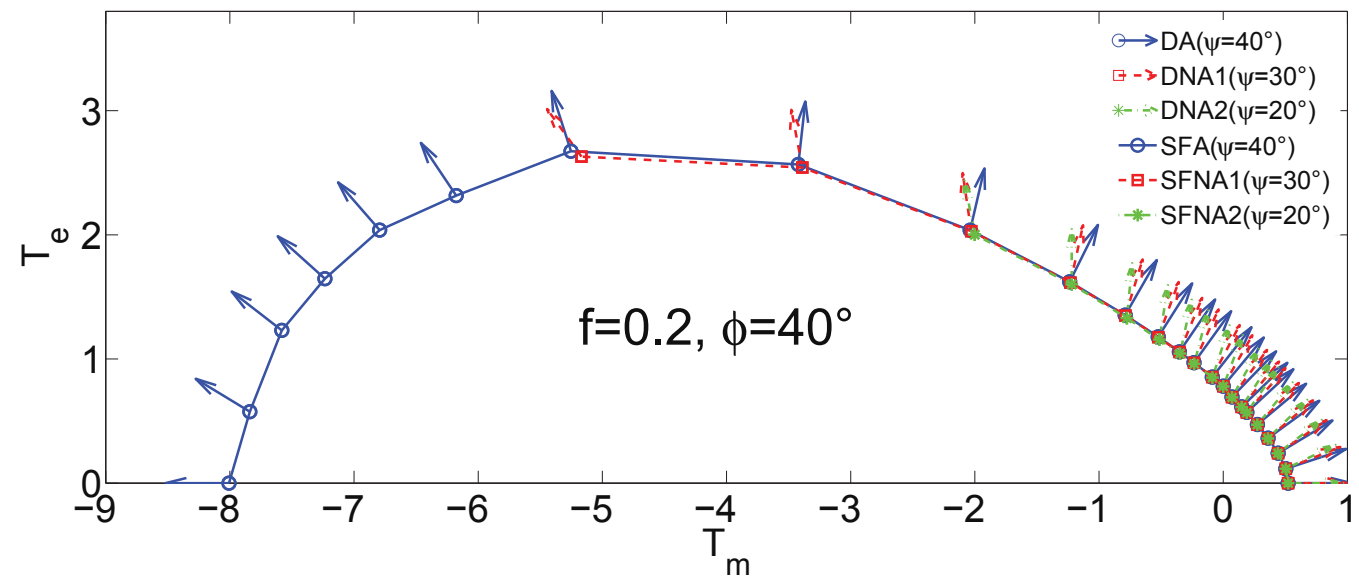

(a) Illustration of FEM results: plastic flow directions (denoted DA for associated case, DNA1 and DNA2 for non-associated ones) and yield surface (denoted SFA for associated case, SFNA1 and SFNA2 for nonassociated ones).

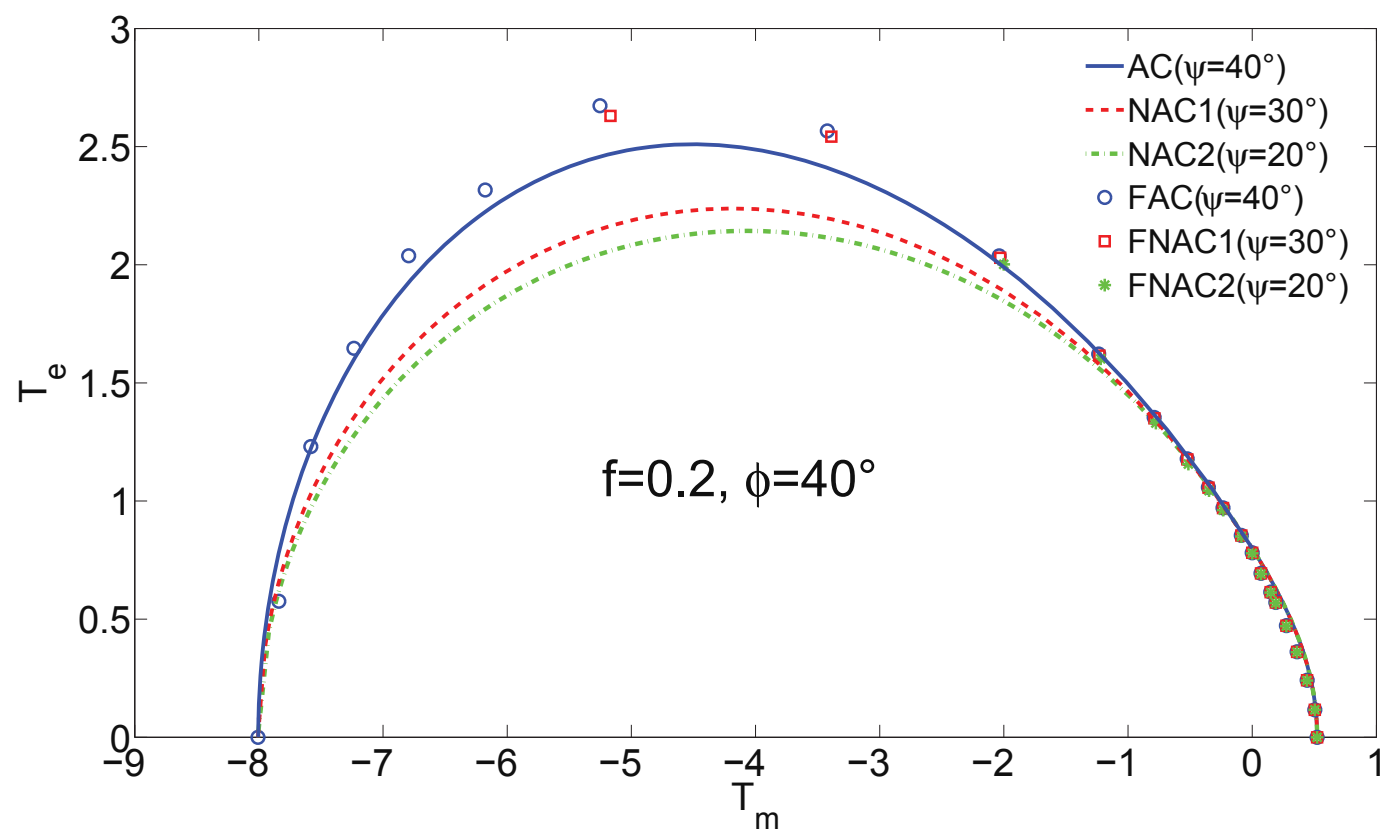

(b) Comparison between the yield surfaces obtained from the established criteria (65) and FEM results (denoted FAC for associated case, FAC1 and FAC2 for non-associated ones).

Figure C.15: Numerical validation for the established model with fixed porosity $f=0.2$ and friction angle $\phi=40^{\circ}$. 


\section{Appendix D. Complementary results concerning the effects of the porosity, friction angle and dilatancy angle on the macroscopic flow rule and on the porosity evolution}

In Figs.D.16 to D.19 we aim to illustrate the influences of porosity $f$ and friction angle $\phi$ on the plastic flow rule with respect to the variation of macroscopic stress triaxiality $T_{m} / T_{e}$, both from the established law (69) and the FEM computations. It can be observed again that the difference between the associated case and the non-associated ones increases with the decrease of the porosity, and with the increase of friction angle. The same conclusion can be deduced from the Figs.D.20-D.23, which illustrate the void growth rate also with repsect to the macroscopic stress triaxiality.

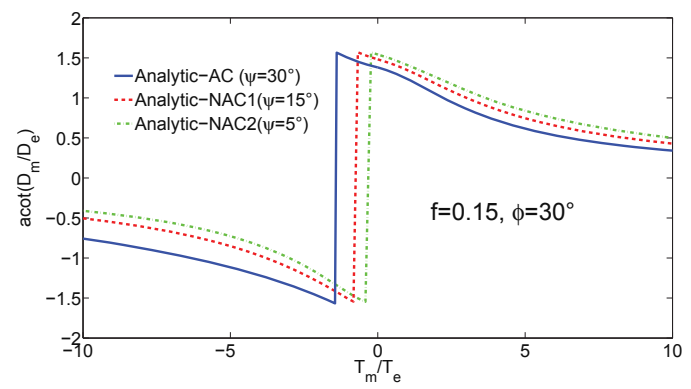

(a) Analytic

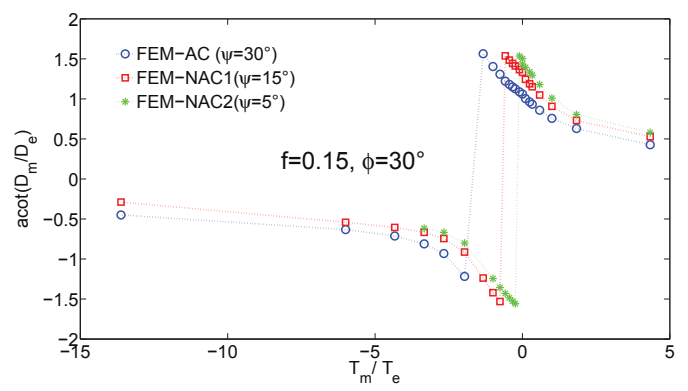

(b) FEM

Figure D.16: Analytical result and FEM solutions for plastic flow direction with the fixed values of porosity $f=0.15$ and friction angle $\phi=30^{\circ}$.

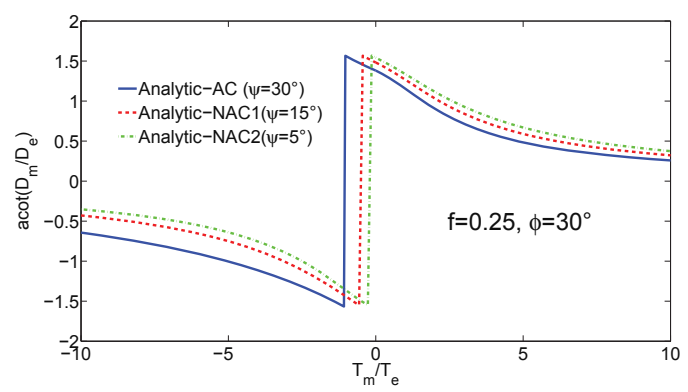

(a) Analytic

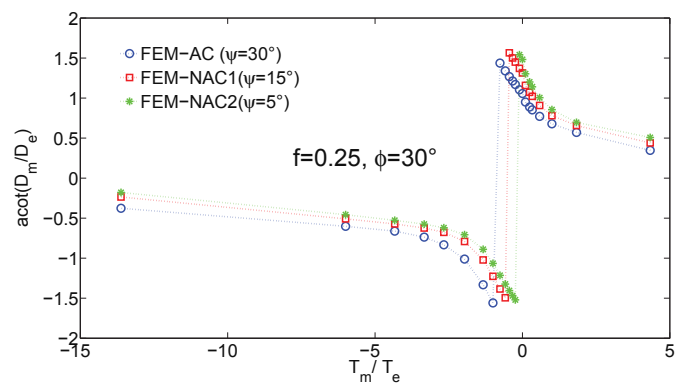

(b) FEM

Figure D.17: Analytical result and FEM solutions for plastic flow direction with the fixed values of porosity $f=0.25$ and friction angle $\phi=30^{\circ}$. 


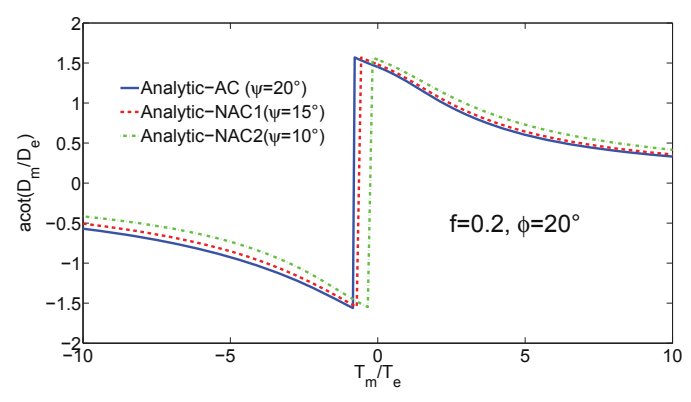

(a) Analytic

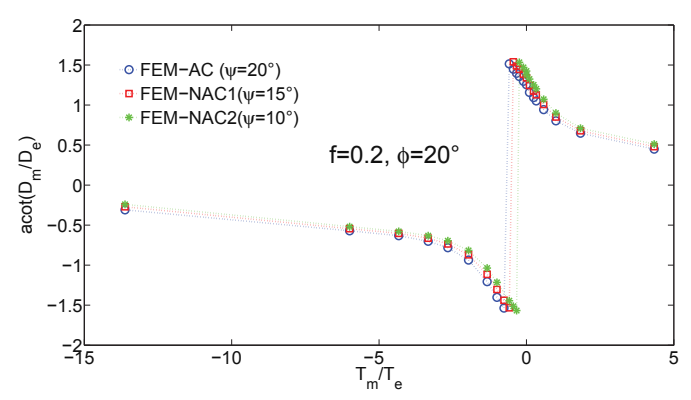

(b) FEM

Figure D.18: Analytical result and FEM solutions for plastic flow direction with the fixed values of porosity $f=0.2$ and friction angle $\phi=20^{\circ}$.

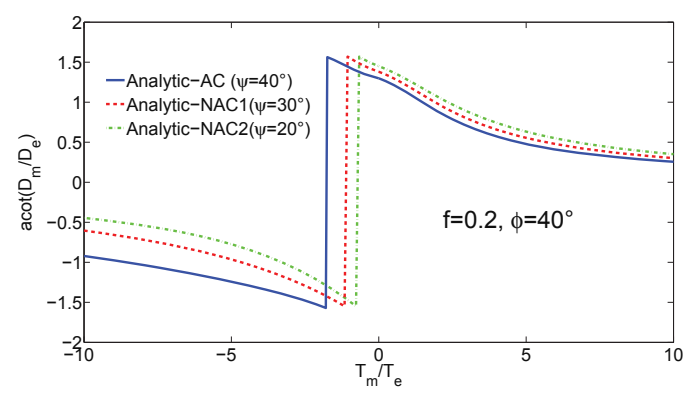

(a) Analytic

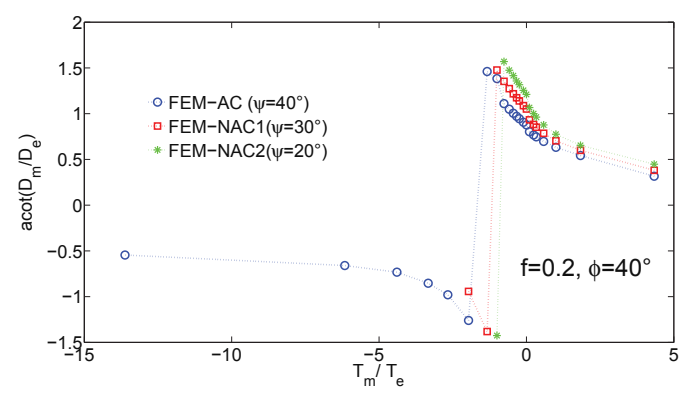

(b) FEM

Figure D.19: Analytical result and FEM solutions for plastic flow direction with the fixed values of porosity $f=0.2$ and friction angle $\phi=40^{\circ}$.

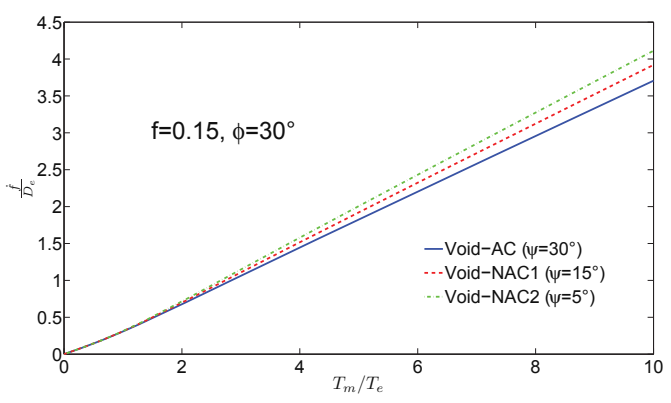

(a) Variation with positive triaxialities

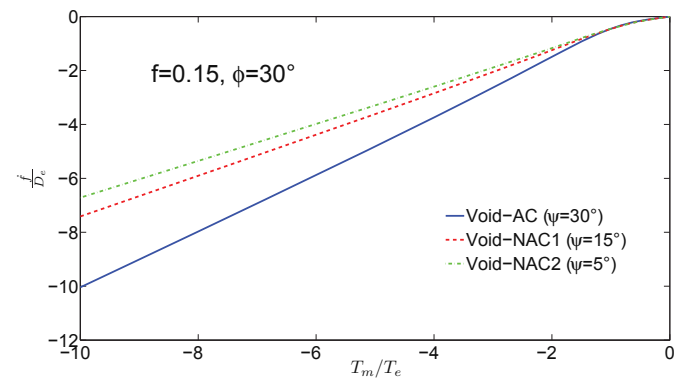

(b) Variation with negative triaxialities

Figure D.20: Analytical result of the plastic void growth with a initial value of porosity $f=0.15$. Friction angle $\phi=30^{\circ}$. 


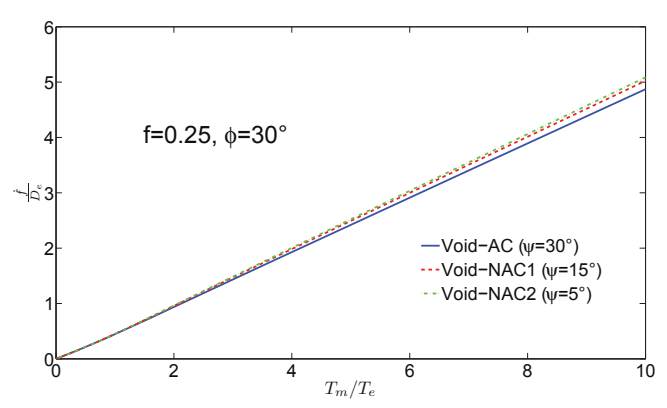

(a) Variation with positive triaxialities

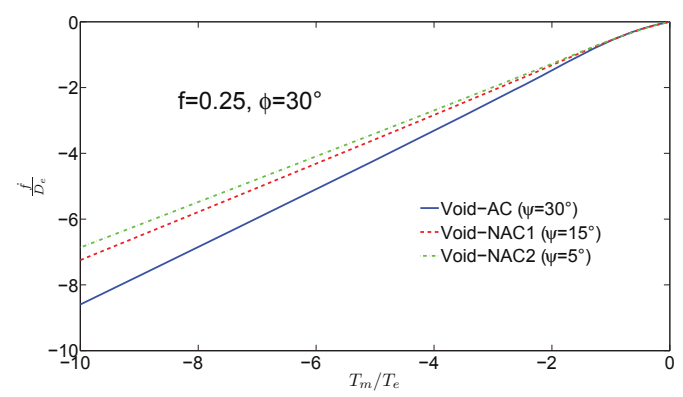

(b) Variation with negative triaxialities

Figure D.21: Analytical result of the plastic void growth with a initial value of porosity $f=0.25$. Friction angle $\phi=30^{\circ}$.

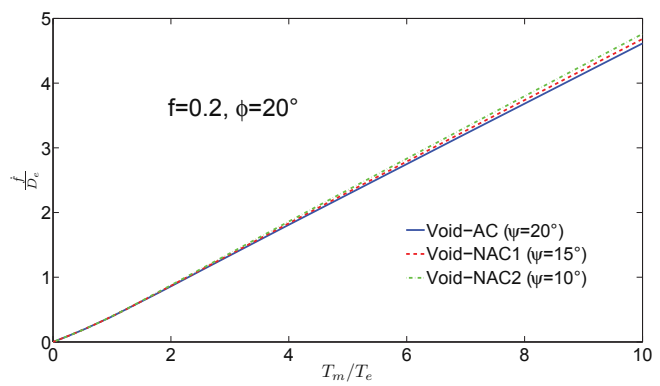

(a) Variation with positive triaxialities

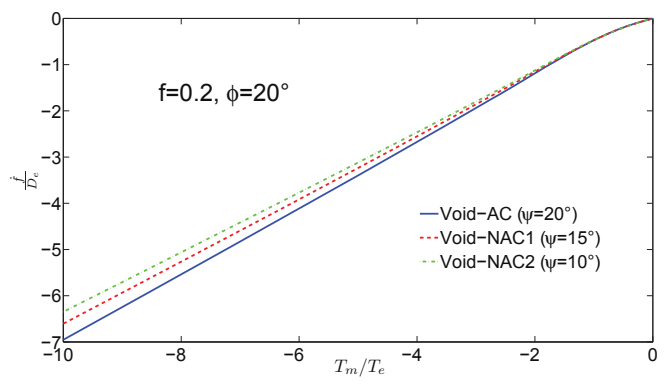

(b) Variation with negative triaxialities

Figure D.22: Analytical result of the plastic void growth with a initial value of porosity $f=0.2$. Friction angle $\phi=20^{\circ}$.

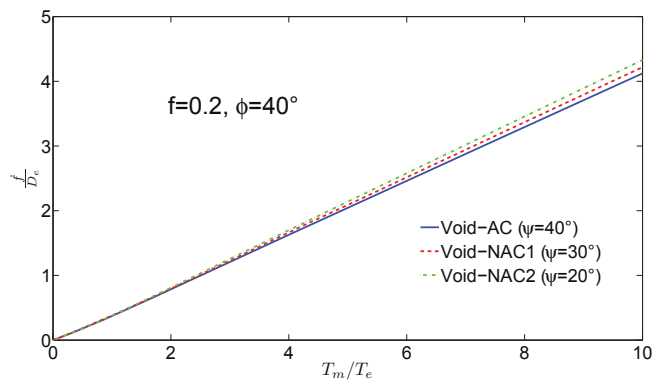

(a) Variation with positive triaxialities

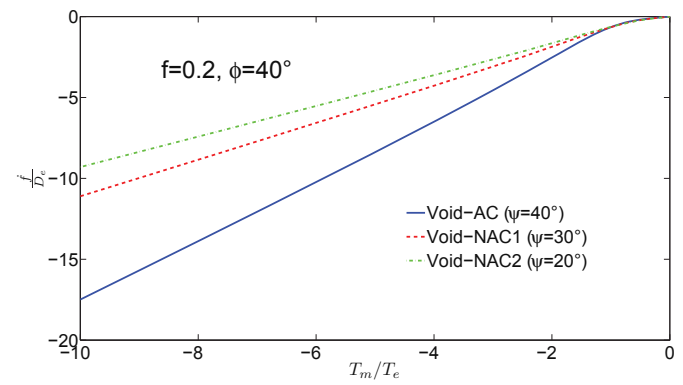

(b) Variation with negative triaxialities

Figure D.23: Analytical result of the plastic void growth with a initial value of porosity $f=0.2$. Friction angle $\phi=40^{\circ}$.. 